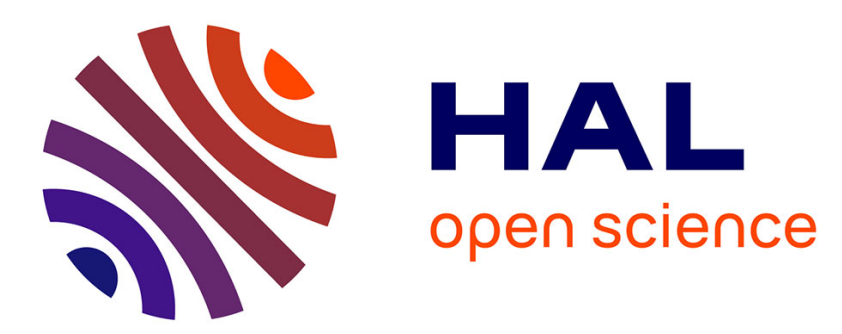

\title{
Reduced models in the medium frequency range for general external structural-acoustics systems
}

\author{
Christian Soize
}

\section{To cite this version:}

Christian Soize. Reduced models in the medium frequency range for general external structuralacoustics systems. Journal of the Acoustical Society of America, 1998, 103 (6), pp.3393-3406. 10.1121/1.423052. hal-00765803

\section{HAL Id: hal-00765803 https://hal.science/hal-00765803}

Submitted on 16 Dec 2012

HAL is a multi-disciplinary open access archive for the deposit and dissemination of scientific research documents, whether they are published or not. The documents may come from teaching and research institutions in France or abroad, or from public or private research centers.
L'archive ouverte pluridisciplinaire HAL, est destinée au dépôt et à la diffusion de documents scientifiques de niveau recherche, publiés ou non, émanant des établissements d'enseignement et de recherche français ou étrangers, des laboratoires publics ou privés. 


\title{
REDUCED MODELS IN THE MEDIUM-FREQUENCY RANGE FOR GENERAL EXTERNAL STRUCTURAL-ACOUSTIC SYSTEMS
}

\author{
Christian Soize
}

Structures Department, ONERA, BP 72, F-92322 Chatillon Cedex, France

Revised Version : 16 February 1998

\begin{abstract}
This paper presents a theoretical approach for constructing a reduced model in the mediumfrequency range in the area of structural acoustics for a general three-dimensional dissipative structure made of an anisotropic, inhomogeneous, viscoelastic bounded medium coupled with an external acoustic fluid. All the results presented can be used if the structure is made of beams, plates and shells. The boundary value problem in the frequency domain and its variational formulation are presented. For a fixed medium-frequency band, an energy operator related to the structural-acoustic system is introduced. This operator is symmetric positive definite and has a countable set of positive eigenvalues. Its dominant eigensubspace allows a reduced model to be constructed using the Ritz-Galerkin method. A finite dimension approximation of the three-dimensional continuous case is presented and an effective construction of the dominant subspace using the subspace iteration method is developed. As an example, the reduced model is used for constructing the time-stationary random response of the structuralacoustic system submitted to a random wall pressure field. Finally, the theory is validated for a finite length circular cylindrical shell coupled with several dashpots and springs and immersed in a gas (air) and in a liquid (water).
\end{abstract}

PACS numbers : $43.40 \mathrm{Gh}$

\section{INTRODUCTION}

This paper is the continuation of initial papers published by the author ${ }^{1,2}$ in the area of modeling and associated solving methods for linear dissipative structural-acoustic systems in the medium-frequency (MF) range. It is known ${ }^{3-6}$ that, for low-frequency (LF) dynamic analysis in structural dynamics, reduced models are a very efficient tool for constructing the 
solution. These techniques correspond to a Ritz-Galerkin reduction of the structural-dynamics model using, for instance, the normal modes corresponding to the lowest eigenfrequencies of the associated conservative structure and called modal reduction. The efficiency of this kind of reduced model is due to the small number of generalized dynamical degrees of freedom used in the representation and in addition, is obtained by solving a well-stated generalized symmetric eigenvalue problem for which only the first eigenvalues and the corresponding eigenfunctions have to be calculated. In addition, when such a reduced model is obtained, responses to deterministic or random excitations can be calculated for no significant additional numerical cost, and the reduced model can be used directly for solving various structural-acoustic problems in the LF range ${ }^{7,8}$. The fundamental problem related to the construction of a reduced model in the MF range for general dissipative structural-acoustic systems has not yet been solved. The author ${ }^{9}$ recently proposed an efficient solution for constructing such a reduced model for general structural-dynamics systems (structures in a vacuum). Here, we present an extension of this theory to the case of general structural-acoustic systems consisting of a structure coupled with an external acoustic fluid (gas or liquid). Concerning the structure, these theoretical developments are presented in the context of three-dimensional viscoelasticity for an arbitrary domain geometry and for an anisotropic, inhomogeneous material. Extension of the results presented to beams, plates and shells is straightforward. Concerning the external acoustic fluid, the geometry of the fluid-structure interface is arbitrary. In Section I, we present the boundary value problem to be solved in the frequency domain and we establish its variational formulation. In Section II, we construct a functional basis adapted to the MF range. To do so, we introduce an energy operator related to the dynamics of the structural-acoustic system in an MF band. This operator is symmetric positive definite and has a countable set of positive eigenvalues. The corresponding eigenfunctions form a complete family in the set of admissible displacement fields of the structure. In Section III, the reduced model is introduced using the Ritz-Galerkin projection of the variational formulation on the dominant eigensubspace of the energy operator, spanned by the eigenfunctions which correspond to the highest eigenvalues. In Section IV, we present the finite dimension approximation of the three-dimensional continuous case allowing the effective calculation to be carried out for the general case. In Section V, we give an efficient procedure for constructing the dominant subspace using the subspace iteration method. To illustrate the interest of such a reduced 
model, Section VI deals with the construction of the time-stationary random response of structural-acoustic systems submitted to a random wall pressure field. Finally, the theory is validated for a finite length circular cylindrical shell coupled with several dashpots and springs and immersed in a gas (Section VII) and in a liquid (Section VIII). The method proposed can also be used in the low-frequency range, but in this case there is no additional gain with respect to the usual modal reduction method. Nevertheless, it should be noted that, for a structure in a vacuum, if the structural damping tends to zero, then it can be proved that the present MF method and the usual modal BF method give the same reduced model in the low-frequency range.

\section{BOUNDARY VALUE PROBLEM AND ITS SOLUTION}

\section{A. Definition of the boundary value problem}

We consider linear vibrations (formulated in the frequency domain $\omega$ ) of a three-dimensional structural-acoustic system around a static equilibrium configuration considered as a natural state at rest (see Fig. 1). Let $\Omega$ be a bounded domain of $\mathbb{R}^{3}$, occupied by the structure at static equilibrium and made of viscoelastic material. Let $\partial \Omega=\Gamma_{0} \cup \Sigma \cup \Gamma$ be the boundary which is assumed to be sufficiently smooth and such that $\Gamma_{0} \cap \Sigma \cap \Gamma=\emptyset$. Let $\mathbf{n}$ be its outward unit normal. Let $\mathbf{u}(\mathbf{x}, \omega)=\left(u_{1}(\mathbf{x}, \omega), u_{2}(\mathbf{x}, \omega), u_{3}(\mathbf{x}, \omega)\right)$ be the displacement field in each point $\mathbf{x}=\left(x_{1}, x_{2}, x_{3}\right)$ in Cartesian coordinates and at frequency $\omega$. On part $\Gamma_{0}$ of the boundary, the structure is fixed $(\mathbf{u}=\mathbf{0})$ whereas on part $\Sigma \cup \Gamma$ it is free. The structure is surrounded by an external inviscid acoustic fluid (gas or liquid) occupying the unbounded three-dimensional domain $\Omega_{E}$ whose boundary $\partial \Omega_{E}$ is $\Gamma$. Let $c_{E}$ be the constant speed of sound and $\rho_{E}$ be the constant mass density of the external fluid at equilibrium. The pressure field and the velocity potential in each point $\mathbf{x}$ in $\Omega_{E}$ and at frequency $\omega$ are denoted as $p(\mathbf{x}, \omega)$ and $\psi(\mathbf{x}, \omega)$ respectively. We introduce a narrow MF band $B$ such that $B=\left[\omega_{B}-\Delta \omega / 2, \omega_{B}+\Delta \omega / 2\right]$ in which $\omega_{B}$ is the center frequency and $\Delta \omega$ is the bandwidth such that $\Delta \omega / \omega_{B} \ll 1$ and $\omega_{B}>\Delta \omega / 2$. With $B$ we associate interval $\widetilde{B}=\left[-\omega_{B}-\Delta \omega / 2,-\omega_{B}+\Delta \omega / 2\right]$. The structure is submitted to a square integrable body force field $\mathbf{x} \mapsto \eta(\omega) \mathbf{f}(\mathbf{x}, \omega)$ from $\Omega$ into $\mathbb{C}^{3}$ and a square integrable surface force field $\mathbf{x} \mapsto \eta(\omega) \mathbf{f}_{\Sigma}(\mathbf{x}, \omega)$ from $\Sigma$ into $\mathbb{C}^{3}$, in which

$\eta(\omega)$ is a function from $\mathbb{R}$ into $\mathbb{C}$, such that $\eta(\omega)=0$ if $\omega$ is not in $B \cup \widetilde{B}$, continuous on $B$, verifying $|\eta(-\omega)|=|\eta(\omega)|$ and such that $|\eta(\omega)| \neq 0$ for all $\omega$ in $B$. Introducing 
components $\left(f_{1}, f_{2}, f_{3}\right)$ and $\left(f_{\Sigma, 1}, f_{\Sigma, 2}, f_{\Sigma, 3}\right)$ of $\mathbf{f}$ and $\mathbf{f}_{\Sigma}$ respectively, the boundary value problem is written as follows in terms of $\mathbf{u}$ and $\psi$ (the convention for the Fourier transform being $\left.v(\omega)=\int_{\mathbb{R}} e^{-i \omega t} v(t) d t\right)$,

$$
\begin{gathered}
-\omega^{2} \rho u_{i}-\sigma_{i j, j}=\eta f_{i} \quad \text { in } \quad \Omega, \\
\sigma_{i j} n_{j}=\eta f_{\Sigma, i} \quad \text { on } \quad \Sigma \quad, \\
\sigma_{i j} n_{j}=-p n_{i} \quad \text { on } \quad \Gamma \quad, \\
u_{i}=0 \quad \text { on } \quad \Gamma_{0}
\end{gathered}
$$

in which $i=1,2,3$ and where the summation over index $j$ is used. Concerning the external acoustic fluid, we have

$$
\begin{gathered}
p=-i \omega \rho_{E} \psi \quad \text { in } \Omega_{E} \cup \Gamma, \\
\nabla^{2} \psi+\frac{\omega^{2}}{c_{E}^{2}} \psi=0 \quad \text { in } \quad \Omega_{E}, \\
\frac{\partial \psi}{\partial \mathbf{n}}=i \omega \mathbf{u} \cdot \mathbf{n} \quad \text { on } \Gamma \quad, \\
|\psi|=O\left(\frac{1}{R}\right) \quad, \quad\left|\frac{\partial \psi}{\partial R}+i \frac{\omega}{c_{E}} \psi\right|=O\left(\frac{1}{R^{2}}\right) \quad \text { as } \quad R=\|\mathbf{x}\| \rightarrow+\infty .
\end{gathered}
$$

In Eq. (1), $\rho(\mathbf{x})>0$ is the mass density of the structure and $\sigma_{i j, j}=\sum_{j=1}^{3} \partial \sigma_{i j} / \partial x_{j}$. For a linear viscoelastic material, stress tensor $\sigma_{i j}$ is written as

$$
\sigma_{i j}=\mathbb{a}_{i j k h}(\mathbf{x}, \omega) \varepsilon_{k h}(\mathbf{u})+\mathfrak{b}_{i j k h}(\mathbf{x}, \omega) \varepsilon_{k h}(i \omega \mathbf{u})
$$

in which the summation over indices $k$ and $h$ is used and where $\varepsilon_{k h}(\mathbf{u})=\left(\partial u_{k} / \partial x_{h}+\right.$ $\left.\partial u_{h} / \partial x_{k}\right) / 2$ is the linearized strain tensor. Coefficients $\mathbb{a}_{i j k h}(\mathbf{x}, \omega)$ and $\mathfrak{b}_{i j k h}(\mathbf{x}, \omega)$ are real, depend on $\mathbf{x}$ and $\omega$, verify the usual properties of symmetry and positiveness ${ }^{10-12}$ and are such that $\mathbb{a}_{i j k h}(\mathbf{x},-\omega)=\mathbb{a}_{i j k h}(\mathbf{x}, \omega)$ and $\mathfrak{b}_{i j k h}(\mathbf{x},-\omega)=\mathfrak{b}_{i j k h}(\mathbf{x}, \omega)$. Eq. (8) is the outward Sommerfeld radiation condition at infinity and $\partial / \partial R$ denotes the derivative in the radial direction from the origin.

\section{B. Acoustic impedance boundary operator and radiation impedance operator}

For all real $\omega \neq 0$ and for a normal velocity field $v=i \omega \mathbf{u} \cdot \mathbf{n}$ given on $\Gamma$, the exterior Neumann problem related to the Helmholtz equation defined by Eqs. (6) to (8) has a unique 
solution ${ }^{13} \psi$. Denoting the values of $\psi$ on surface $\Gamma$ as $\psi_{\Gamma}$, the corresponding pressure field $p_{\Gamma}=-i \omega \rho_{E} \psi_{\Gamma}$ on $\Gamma$ can be written as

$$
p_{\Gamma}=\mathbf{Z}_{\Gamma}(\omega)\{i \omega \mathbf{u} \cdot \mathbf{n}\}
$$

in which $\mathbf{Z}_{\Gamma}(\omega)$ is the acoustic impedance boundary operator related to surface $\Gamma$. At any point $\mathbf{x}$ in unbounded domain $\Omega_{E}$, pressure $p(\mathbf{x}, \omega)$ can be written as

$$
p(\mathbf{x}, \omega)=\mathbf{Z}_{r a d}(\mathbf{x}, \omega)\{i \omega \mathbf{u} \cdot \mathbf{n}\} \quad,
$$

in which $\mathbf{Z}_{\text {rad }}(\mathbf{x}, \omega)$ is the radiation impedance operator. For simply shaped geometries of $\Gamma$ such as baffled rectangular or circular plates, baffled circular cylinder, etc, analytical methods ${ }^{14}$ can be used for constructing operators $\mathbf{Z}_{\Gamma}(\omega)$ and $\mathbf{Z}_{\text {rad }}(\mathbf{x}, \omega)$. For an arbitrary shaped surface $\Gamma$, boundary element methods can be used. This numerical method must be based on the use of integral equation techniques overcoming the mathematical difficulty induced by the problem of spurious or irregular frequencies ${ }^{8,15-17}$.

\section{Variational formulation}

For the general methodology concerning the construction of a variational formulation of a boundary value problem, we refer the reader to Refs. 13, 18 and 19. Let $H$ be the space of all the square integrable functions on $\Omega$ with values in $\mathbb{C}^{3}$, equipped with the inner product $(\mathbf{u}, \mathbf{v})_{H}=\int_{\Omega} \mathbf{u}(\mathbf{x}) \cdot \overline{\mathbf{v}(\mathbf{x})} d \mathbf{x}$ and the associated norm $\|\mathbf{u}\|_{H}=(\mathbf{u}, \mathbf{u})_{H}^{1 / 2}$, in which $\bar{z}$ denotes the conjugate of the complex number $z$. Let $V \subset H$ be the space of admissible displacement fields $\mathbf{u}$ defined on $\Omega$ with values in $\mathbb{C}^{3}$ such that $\mathbf{u}=\mathbf{0}$ on $\Gamma_{0}$ and such that $\nabla u_{k} \in H$ for $k=1,2,3$. The variational formulation of the boundary value problem in $\mathbf{u}$ defined by Eqs. (1) to (9) can be expressed as follows. For all $\omega$ in $B \cup \widetilde{B}$, find $\mathbf{u}(\omega)$ in $V$ such that

$$
a(\mathbf{u}, \mathbf{v} ; \omega)=f(\mathbf{v} ; \omega) \quad, \quad \forall \mathbf{v} \in V \quad
$$

in which $f(\mathbf{v} ; \omega)$ is defined by

$$
f(\mathbf{v} ; \omega)=\eta(\omega)\left\{\int_{\Sigma} \mathbf{f}_{\Sigma}(\mathbf{x}, \omega) \cdot \overline{\mathbf{v}(\mathbf{x})} d s(\mathbf{x})+\int_{\Omega} \mathbf{f}(\mathbf{x}, \omega) \cdot \overline{\mathbf{v}(\mathbf{x})} d \mathbf{x}\right\}
$$

and where $a(\mathbf{u}, \mathbf{v} ; \omega)$ is written as

$$
a(\mathbf{u}, \mathbf{v} ; \omega)=-\omega^{2}\left(m(\mathbf{u}, \mathbf{v})+b_{\Gamma}(\mathbf{u}, \mathbf{v} ; \omega)\right)+i \omega d(\mathbf{u}, \mathbf{v} ; \omega)+k(\mathbf{u}, \mathbf{v} ; \omega)
$$




$$
\begin{gathered}
m(\mathbf{u}, \mathbf{v})=\int_{\Omega} \rho(\mathbf{x}) \mathbf{u}(\mathbf{x}) \cdot \overline{\mathbf{v}(\mathbf{x})} d \mathbf{x}, \\
d(\mathbf{u}, \mathbf{v} ; \omega)=\int_{\Omega} \mathfrak{b}_{i j k h}(\mathbf{x}, \omega) \varepsilon_{k h}(\mathbf{u}) \varepsilon_{i j}(\overline{\mathbf{v}}) d \mathbf{x}, \\
k(\mathbf{u}, \mathbf{v} ; \omega)=\int_{\Omega} \mathbb{a}_{i j k h}(\mathbf{x}, \omega) \varepsilon_{k h}(\mathbf{u}) \varepsilon_{i j}(\overline{\mathbf{v}}) d \mathbf{x},
\end{gathered}
$$

in which the summation over indices $i, j, k$ and $h$ is used. It is assumed that $d(\mathbf{u}, \mathbf{v} ; \omega)$ and $k(\mathbf{u}, \mathbf{v} ; \omega)$ are continuous functions on band $B$ with respect to $\omega$. Finally, $b_{\Gamma}(\mathbf{u}, \mathbf{v} ; \omega)$ is such that

$$
-\omega^{2} b_{\Gamma}(\mathbf{u}, \mathbf{v} ; \omega)=i \omega \int_{\Gamma}(\overline{\mathbf{v}} \cdot \mathbf{n}) \mathbf{Z}_{\Gamma}(\omega)\{\mathbf{u} \cdot \mathbf{n}\} d s
$$

For all $\omega$ in $B \cup \widetilde{B}$, Eq. (12) has a unique solution $\mathbf{u}(\omega)$ in $V$.

\section{CONSTRUCTION OF A FUNCTIONAL BASIS FOR THE REDUCED MODEL}

In order to construct a functional basis adapted to MF band $B$ and useful for the Ritz-Galerkin method, we begin by introducing the operator-valued frequency response function defined for a special class of mechanical excitations. In a second step, we define the energy operator and we construct the functional basis as its eigenfunctions.

\section{A. Operator-valued frequency response function}

Let us assumed that $\mathbf{f}_{\Sigma}=\mathbf{0}$ and that $\mathbf{f}$ is independent of $\omega$. This assumption is only used for constructing the functional basis. As soon as the reduced model is obtained, the response of the mechanical system can be calculated for any deterministic and random excitations which do not use this restrictive assumption (see Eq. (13), Sections III and IV). Then Eq. (13) yields $f(\mathbf{v} ; \omega)=\eta(\omega)(\mathbf{f}, \mathbf{v})_{H}$. It is proved ${ }^{1,2,9}$ that for all $\omega$ fixed in $B \cup \widetilde{B}$, Eq. (12) has a unique solution $\mathbf{u}^{\mathbf{f}}(\omega)$ belonging to $V \subset H$ which can be written as

$$
\mathbf{u}^{\mathbf{f}}(\omega)=\eta(\omega) \mathbf{T}_{H}(\omega) \mathbf{f}
$$

where $\mathbf{T}_{H}(\omega)$ is an operator in $H$. Solution $\mathbf{u}^{\mathbf{f}}(\omega)$ corresponds to the vibration induced by excitation $\eta(\omega) \mathbf{f}$ and $\mathbf{T}_{H}(\omega)$ is the operator-valued frequency response function.

\section{B. Definition of an energy operator}

The energy $\varepsilon_{B}\left(\mathbf{u}^{\mathbf{f}}\right)$ of a structural vibration corresponding to $\mathbf{u}^{\mathbf{f}}$ given by Eq. (15) is defined as twice the value of the total kinetic energy of the structure, i.e. using the Plancherel formula,

$$
\varepsilon_{B}\left(\mathbf{u}^{\mathbf{f}}\right)=\frac{1}{2 \pi} \int_{B \cup \widetilde{B}} \omega^{2}\left(\mathbf{M} \mathbf{u}^{\mathbf{f}}(\omega), \mathbf{u}^{\mathbf{f}}(\omega)\right)_{H} d \omega,
$$


in which $\mathbf{M}$ is the mass operator in $H$ such that $(\mathbf{M} \mathbf{u}, \mathbf{v})_{H}=m(\mathbf{u}, \mathbf{v})$. Let $\mathbf{u}^{\mathbf{f}}(\omega)=$ $\eta(\omega) \mathbf{T}_{H}(\omega) \mathbf{f}$ and $\mathbf{u}^{\mathbf{g}}(\omega)=\eta(\omega) \mathbf{T}_{H}(\omega) \mathbf{g}$ be the solutions corresponding to the vibrations induced by excitations $\eta(\omega) \mathbf{f}$ and $\eta(\omega) \mathbf{g}$ respectively, where $\mathbf{f}$ and $\mathbf{g}$ are in $H$ and are independent of $\omega$. The energy operator $\mathbf{E}_{B}$ relative to band $B$ is defined by

$$
\left(\mathbf{E}_{B} \mathbf{f}, \mathbf{g}\right)_{H}=\frac{1}{2 \pi} \int_{B \cup \widetilde{B}} \omega^{2}\left(\mathbf{M} \mathbf{u}^{\mathbf{f}}(\omega), \mathbf{u}^{\mathbf{g}}(\omega)\right)_{H} d \omega
$$

We then deduce that $\varepsilon_{B}\left(\mathbf{u}^{\mathbf{f}}\right)=\left(\mathbf{E}_{B} \mathbf{f}, \mathbf{f}\right)_{H}$. It should be noted that operator $\mathbf{E}_{B}$ depends on $B$ and $\eta$ but does not depend on spatial parts $\mathbf{f}$ and $\mathbf{g}$ of the excitations. In addition, this operator depends on the external acoustic fluid due to the coupling operator. In the present context, operator $\mathbf{E}_{B}$ is only used to calculate a functional basis for constructing a reduced model of the structure coupled with the external acoustic fluid. In Section VII which is devoted to the coupling with an external liquid, we will see that the coupling operator must be kept for the calculation of $\mathbf{E}_{B}$. A contrario, the coupling operator can be omitted for the calculation of $\mathbf{E}_{B}$ in the case of a coupling with a gas as shown in Section VIII.

\section{Properties of the energy operator}

Energy operator $\mathbf{E}_{B}$ is a positive-definite symmetric operator in $H$ whose range space is a subspace of $V$ and which can be written as

$$
\mathbf{E}_{B}=\frac{1}{\pi} \int_{B} \omega^{2}|\eta(\omega)|^{2} \Re e\left\{\mathbf{T}_{H}(\omega)^{*} \mathbf{M} \mathbf{T}_{H}(\omega)\right\} d \omega
$$

in which $\Re e$ denotes the real part and where $\mathbf{T}_{H}(\omega)^{*}$ is the adjoint of $\mathbf{T}_{H}(\omega)$. Consequently, operator $\mathbf{E}_{B}$ has a countable set of decreasing positive eigenvalues with finite multiplicity, possibly excepting zero, $\lambda_{1} \geq \lambda_{2} \geq \ldots \rightarrow 0$, in which the $\lambda_{\nu}$ terms are the repeated eigenvalues of $\mathbf{E}_{B}$. The corresponding eigenfunctions $\left\{\mathbf{e}_{\nu}\right\}_{\nu \geq 1}$ such that

$$
\mathbf{E}_{B} \mathbf{e}_{\nu}=\lambda_{\nu} \mathbf{e}_{\nu}
$$

are functions $\mathbf{e}_{\nu}(\mathbf{x})$ from $\Omega$ into $\mathbb{R}^{3}$ and form a complete family in admissible space $V \subset H$, orthonormal for the inner product of $H$. This fundamental result allows a reduced model to be constructed in the MF range. We then have $\left(\mathbf{e}_{\nu}, \mathbf{e}_{\nu^{\prime}}\right)_{H}=\delta_{\nu \nu^{\prime}}$. Finally, the eigenvalues are such that $\sum_{\nu=1}^{+\infty} \lambda_{\nu}<+\infty$ (one says that $\mathbf{E}_{B}$ is a trace operator ${ }^{20,21}$ ) and its trace norm denoted as $\operatorname{tr} \mathbf{E}_{B}$ is written as

$$
\operatorname{tr} \mathbf{E}_{B}=\sum_{\nu=1}^{+\infty} \lambda_{\nu}<+\infty
$$


The proof of these results is a straightforward extension of the proof established by the author ${ }^{9}$ for the case of a three-dimensional viscoelastic structure in a vacuum.

\section{CONSTRUCTION OF A REDUCED MODEL IN THE MF RANGE}

The reduced model adapted to MF band $B$ is obtained using the Ritz-Galerkin projection of the variational formulation defined by Eq. (12) on the subspace $V^{N}$ of $V$ spanned by the eigenfunctions $\left\{\mathbf{e}_{1}, \ldots, \mathbf{e}_{N}\right\}$ corresponding to the $N$ highest eigenvalues $\left\{\lambda_{1}, \ldots, \lambda_{N}\right\}$ of energy operator $\mathbf{E}_{B}$. Let $\mathbf{u}(\omega) \in V$ be the solution of Eq. (12) and let $\mathbf{u}^{N}(\omega)$ be its projection on $V^{N}$,

$$
\mathbf{u}^{N}(\mathbf{x}, \omega)=\sum_{\nu=1}^{N} \theta_{\nu}(\omega) \mathbf{e}_{\nu}(\mathbf{x})
$$

in which $\theta_{\nu}(\omega) \in \mathbb{C}$. From Eq. (12), we deduce that, for all $\omega$ in $B \cup \widetilde{B}, \boldsymbol{\theta}(\omega)=$ $\left(\theta_{1}(\omega), \ldots, \theta_{N}(\omega)\right) \in \mathbb{C}^{N}$ is the unique solution of the linear equation

$$
\left[\mathcal{A}_{N}(\omega)\right] \boldsymbol{\theta}(\omega)=\eta(\omega) \mathcal{F}(\omega)
$$

in which $\left[\mathcal{A}_{N}(\omega)\right]$ is the symmetric $(N \times N)$ complex matrix defined by $\left[\mathcal{A}_{N}(\omega)\right]_{\nu \nu^{\prime}}=$ $a\left(\mathbf{e}_{\nu^{\prime}}, \mathbf{e}_{\nu} ; \omega\right)$ and where $\mathcal{F}(\omega)=\left(\mathcal{F}_{1}(\omega), \ldots, \mathcal{F}_{N}(\omega)\right) \in \mathbb{C}^{N}$ is such that $\eta(\omega) \mathcal{F}_{\nu}(\omega)=$ $f\left(\mathbf{e}_{\nu} ; \omega\right)$. Eqs. (21) and (22) constitute the reduced model in MF band $B$ of the structuralacoustic dynamical system described by Eqs. (1) to (9). For all $\omega$ in $B \cup \widetilde{B}$, matrix $\left[\mathcal{A}_{N}(\omega)\right]$ is invertible and the solution of Eq. (22) is written as $\boldsymbol{\theta}(\omega)=\eta(\omega)\left[\mathcal{T}_{N}(\omega)\right] \mathcal{F}(\omega)$ in which $\left[\mathcal{T}_{N}(\omega)\right]=\left[\mathcal{A}_{N}(\omega)\right]^{-1}$. For all $\omega$ in $B \cup \widetilde{B}, \mathbf{u}^{N}(\omega) \rightarrow \mathbf{u}(\omega)$ in $V$ as $N \rightarrow+\infty$.

\section{FINITE DIMENSION APPROXIMATION}

An explicit construction of eigenfunctions $\left\{\mathbf{e}_{1}, \ldots, \mathbf{e}_{N}\right\}$ of energy operator $\mathbf{E}_{B}$ cannot be obtained in the general case. A finite dimension approximation $\mathbf{E}_{B, n}$ of $\mathbf{E}_{B}$ must be introduced and the eigenfunctions $\left\{\mathbf{e}_{1}^{n} \ldots, \mathbf{e}_{N}^{n}\right\}$ of $\mathbf{E}_{B, n}$ associated with the $N$ highest eigenvalues $\lambda_{1}^{n} \geq \ldots \geq \lambda_{N}^{n}$, are the approximation of $\left\{\mathbf{e}_{1} \ldots, \mathbf{e}_{N}\right\}$. This finite approximation is obtained by using the Ritz-Galerkin method. We then consider a subspace $V_{n} \subset V$ of finite dimension $n \geq 1$, spanned by a family $\left\{\boldsymbol{\psi}_{1}, \ldots, \boldsymbol{\psi}_{n}\right\}$ of independent $\mathbb{R}^{3}$-valued functions $\left\{\boldsymbol{\psi}_{\alpha}\right\}_{\alpha}$ in $V$. In practice, this family can be either a finite element basis ${ }^{13,22-23}$ associated with a finite element mesh of domain $\Omega$ or, for a particular case, any functional basis constructed in the context of an analytical approach, for instance the sequence of the structural normal modes of 
a homogeneous, simply supported rectangular plate with a constant thickness. Let $\mathbf{u}(\omega) \in V$ be the solution of Eq. (12). Its projection on $V_{n}$ is written as

$$
\mathbf{u}_{n}(\mathbf{x}, \omega)=\sum_{\alpha=1}^{n} q_{\alpha}(\omega) \boldsymbol{\psi}_{\alpha}(\mathbf{x})
$$

From Eq. (12), we deduce that $\mathbf{q}(\omega)=\left(q_{1}(\omega), \ldots, q_{n}(\omega)\right) \in \mathbb{C}^{n}$ is the unique solution of the linear equation

$$
\left[A_{n}(\omega)\right] \mathbf{q}(\omega)=\eta(\omega) \mathbf{F}(\omega)
$$

in which symmetric $(n \times n)$ complex matrix $\left[A_{n}(\omega)\right]$ is such that $\left[A_{n}(\omega)\right]_{\alpha \beta}=a\left(\boldsymbol{\psi}_{\beta}, \boldsymbol{\psi}_{\alpha} ; \omega\right)$ and can be written as

$$
\left[A_{n}(\omega)\right]=-\omega^{2}\left([M]+\left[B_{\Gamma}(\omega)\right]\right)+i \omega[D(\omega)]+[K(\omega)]
$$

in which $[M],[D(\omega)]$ and $[K(\omega)]$ are positive-definite symmetric $(n \times n)$ real matrices such that $[M]_{\alpha \beta}=m\left(\boldsymbol{\psi}_{\beta}, \boldsymbol{\psi}_{\alpha}\right),[D(\omega)]_{\alpha \beta}=d\left(\boldsymbol{\psi}_{\beta}, \boldsymbol{\psi}_{\alpha} ; \omega\right)$ and $[K(\omega)]_{\alpha \beta}=k\left(\boldsymbol{\psi}_{\beta}, \boldsymbol{\psi}_{\alpha} ; \omega\right)$, and $\left[B_{\Gamma}(\omega)\right]$ is a symmetric $(n \times n)$ complex matrix such that $\left[B_{\Gamma}(\omega)\right]_{\alpha \beta}=b_{\Gamma}\left(\boldsymbol{\psi}_{\beta}, \boldsymbol{\psi}_{\alpha} ; \omega\right)$. Vector $\mathbf{F}(\omega)=\left(F_{1}(\omega), \ldots, F_{n}(\omega)\right) \in \mathbb{C}^{n}$ is such that

$$
\eta(\omega) F_{\alpha}(\omega)=f\left(\boldsymbol{\psi}_{\alpha} ; \omega\right)
$$

The pressure field radiated by the structure is written as

$$
p_{n}(\mathbf{x}, \omega)=i \omega \sum_{\alpha=1}^{n} Z_{\alpha}^{n}(\mathbf{x}, \omega) q_{\alpha}(\omega)
$$

in which $Z_{\alpha}^{n}(\mathbf{x}, \omega)=\mathbf{Z}_{\text {rad }}(\mathbf{x}, \omega)\left\{\boldsymbol{\psi}_{\alpha} \cdot \mathbf{n}\right\}$. Concerning the far field radiated by the structure, we introduce the spherical coordinates

$$
\mathbf{x}=\mathbf{x}(R, \theta, \varphi)=(R \sin \varphi \cos \theta, R \sin \varphi \sin \theta, R \cos \varphi)
$$

with $\varphi \in[0, \pi]$ and $\theta \in[0,2 \pi]$. The far field can then be characterized by the pressure coefficient $c^{n}(\theta, \varphi, \omega)$ defined by

$$
c^{n}(\theta, \varphi, \omega)=\lim _{R \rightarrow+\infty}\left\{\frac{R e^{i \omega R / c_{E}}}{\rho_{E} c_{E}^{2}} p_{n}(\mathbf{x}(R, \theta, \varphi), \omega)\right\} .
$$

Substituting Eq. (27) in Eq. (29) yields

$$
c^{n}(\theta, \varphi, \omega)=i \omega \mathbf{r}^{n}(\theta, \varphi, \omega)^{T} \mathbf{q}(\omega)=i \omega \sum_{\alpha=1}^{n} r_{\alpha}^{n}(\theta, \varphi, \omega) q_{\alpha}(\omega)
$$


in which $\mathbf{r}^{n}(\theta, \varphi, \omega)=\left(r_{1}^{n}(\theta, \varphi, \omega), \ldots, r_{n}^{n}(\theta, \varphi, \omega)\right) \in \mathbb{C}^{n}$ and where $r_{\alpha}(\theta, \varphi, \omega)$ is such that

$$
r_{\alpha}^{n}(\theta, \varphi, \omega)=\lim _{R \rightarrow+\infty}\left\{\frac{R e^{i \omega R / c_{E}}}{\rho_{E} c_{E}^{2}} Z_{\alpha}^{n}(\mathbf{x}(R, \theta, \varphi), \omega)\right\} .
$$

\section{A. Projection of the energy operator}

Projection $\mathbf{E}_{B, n}$ of operator $\mathbf{E}_{B}$ on $V_{n} \subset H$ is written as

$$
\mathbf{E}_{B, n}=\sum_{\alpha, \beta=1}^{n}\left[E_{n}\right]_{\alpha \beta}\left(., \boldsymbol{\psi}_{\beta}\right)_{H} \boldsymbol{\psi}_{\alpha}
$$

in which $\left[E_{n}\right]$ is a positive-definite symmetric $(n \times n)$ real matrix such that

$$
\begin{gathered}
{\left[E_{n}\right]=\int_{B}\left[e_{n}(\omega)\right] d \omega,} \\
{\left[e_{n}(\omega)\right]=\frac{1}{\pi} \omega^{2}|\eta(\omega)|^{2} \Re e\left\{\left[T_{n}(\omega)\right]^{*}[M]\left[T_{n}(\omega)\right]\right\}}
\end{gathered}
$$

where $\left[T_{n}(\omega)\right]$ is the symmetric $(n \times n)$ complex matrix such that

$$
\left[T_{n}(\omega)\right]=\left[A_{n}(\omega)\right]^{-1} \quad, \quad\left[T_{n}(\omega)\right]^{*}=\overline{\left[T_{n}(\omega)\right]}
$$

\section{B. Generalized symmetric eigenvalue problem}

Each eigenfunction $\mathbf{e}_{\nu}^{n}$ such that $\mathbf{E}_{B, n} \mathbf{e}_{\nu}^{n}=\lambda_{\nu}^{n} \mathbf{e}_{\nu}^{n}$ can be written as

$$
\mathbf{e}_{\nu}^{n}=\sum_{\alpha=1}^{n} P_{\alpha}^{\nu} \boldsymbol{\psi}_{\alpha}
$$

Introducing vector $\mathbf{P}^{\nu}=\left(P_{1}^{\nu}, \ldots, P_{n}^{\nu}\right)$, it can easily be seen that eigenvalues $\left\{\lambda_{1}^{n}, \ldots, \lambda_{n}^{n}\right\}$ and the corresponding eigenvectors $\left\{\mathbf{P}^{1}, \ldots, \mathbf{P}^{n}\right\}$ are the solutions of the generalized symmetric eigenvalue problem

$$
[H] \mathbf{P}=\lambda^{n}[G] \mathbf{P}
$$

in which $[G]$ and $[H]$ are positive-definite symmetric $(n \times n)$ real matrices such that $[G]_{\beta \alpha}=$ $\left(\boldsymbol{\psi}_{\alpha}, \boldsymbol{\psi}_{\beta}\right)_{H}$ and $[H]=[G]\left[E_{n}\right][G]$. Eigenvectors $\left\{\mathbf{P}^{1}, \ldots, \mathbf{P}^{n}\right\}$ form a basis of $\mathbb{R}^{n}$ and verify the usual orthogonality conditions with respect to $[G]$ and $[H]$ and consequently, we have $\left(\mathbf{e}_{\nu}^{n}, \mathbf{e}_{\nu^{\prime}}^{n}\right)_{H}=\left([G] \mathbf{P}^{\nu}, \mathbf{P}^{\nu^{\prime}}\right)_{\mathbb{R}^{n}}=\delta_{\nu \nu^{\prime}}$.

\section{Reduced model adapted to MF band $B$}

J. Acoust. Soc. Am. 
Let $N<n$ (generally $N \ll n$ ). Let $\mathbf{u}(\omega) \in V$ be the solution of Eq. (12). The reduced model adapted to MF band $B$ is defined as the projection $\mathbf{u}_{n}^{N}(\omega)$ of $\mathbf{u}(\omega)$ on the subspace $V_{n}^{N} \subset V_{n} \subset V$ spanned by the eigenfunctions $\left\{\mathbf{e}_{1}^{n}, \ldots, \mathbf{e}_{N}^{n}\right\}$ which correspond to the $N$ highest eigenvalues $\lambda_{1}^{n} \geq \lambda_{2}^{n} \geq \ldots \geq \lambda_{N}^{n}$ of operator $\mathbf{E}_{B, n}$. Projection $\mathbf{u}_{n}^{N}(\omega)$ can be written as

$$
\mathbf{u}_{n}^{N}(\mathbf{x}, \omega)=\sum_{\nu=1}^{N} \theta_{\nu}^{n}(\omega) \mathbf{e}_{\nu}^{n}(\mathbf{x})
$$

in which $\boldsymbol{\theta}^{n}(\omega)=\left(\theta_{1}^{n}(\omega), \ldots, \theta_{N}^{n}(\omega)\right) \in \mathbb{C}^{N}$ is the solution of the linear equation

$$
\left[\mathcal{A}_{N}^{n}(\omega)\right] \boldsymbol{\theta}^{n}(\omega)=\eta(\omega) \mathcal{F}^{n}(\omega)
$$

where $\left[\mathcal{A}_{N}^{n}(\omega)\right]$ is the symmetric $(N \times N)$ complex matrix defined by $\left[\mathcal{A}_{N}^{n}(\omega)\right]_{\nu \nu^{\prime}}=$ $a\left(\mathbf{e}_{\nu^{\prime}}^{n}, \mathbf{e}_{\nu}^{n} ; \omega\right)$ and where $\mathcal{F}^{n}(\omega)=\left(\mathcal{F}_{1}^{n}(\omega), \ldots, \mathcal{F}_{N}^{n}(\omega)\right) \in \mathbb{C}^{N}$ is such that $\eta(\omega) \mathcal{F}_{\nu}^{n}(\omega)=$ $f\left(\mathbf{e}_{\nu}^{n} ; \omega\right)$. Matrix $\left[\mathcal{A}_{N}^{n}(\omega)\right]$ is invertible and we have

$$
\boldsymbol{\theta}^{n}(\omega)=\eta(\omega)\left[\mathcal{T}_{N}^{n}(\omega)\right] \mathcal{F}^{n}(\omega) \quad ; \quad\left[\mathcal{T}_{N}^{n}(\omega)\right]=\left[\mathcal{A}_{N}^{n}(\omega)\right]^{-1}
$$

The pressure field radiated by the structure is written as

$$
p_{n}^{N}(\mathbf{x}, \omega)=i \omega \sum_{\nu=1}^{N} Z_{N, \nu}^{n}(\mathbf{x}, \omega) \theta_{\nu}^{n}(\omega)
$$

in which $Z_{N, \nu}^{n}(\mathbf{x}, \omega)=\mathbf{Z}_{\text {rad }}(\mathbf{x}, \omega)\left\{\mathbf{e}_{\nu}^{n} \cdot \mathbf{n}\right\}$. For this reduced model, the pressure coefficient defined by Eq. (29) and given by Eq. (30) is written as

$$
c_{N}^{n}(\theta, \varphi, \omega)=i \omega \mathbf{r}_{N}^{n}(\theta, \varphi, \omega)^{T} \boldsymbol{\theta}^{n}(\omega)
$$

in which complex vector $\mathbf{r}_{N}^{n}(\theta, \varphi, \omega) \in \mathbb{C}^{N}$ is such that

$$
r_{N, \nu}^{n}(\theta, \varphi, \omega)=\lim _{R \rightarrow+\infty}\left\{\frac{R e^{i \omega R / c_{E}}}{\rho_{E} c_{E}^{2}} Z_{N, \nu}^{n}(\mathbf{x}(R, \theta, \varphi), \omega)\right\}
$$

Let $[P]$ be the $(n \times N)$ real matrix whose columns are the $N$ eigenvectors $\left\{\mathbf{P}^{1}, \ldots, \mathbf{P}^{N}\right\}$ corresponding to the $N$ highest eigenvalues $\lambda_{1}^{n} \geq \ldots \geq \lambda_{N}^{n}$. We then deduce that

$$
\begin{gathered}
\mathbf{q}(\omega)=[P] \boldsymbol{\theta}^{n}(\omega), \\
{\left[\mathcal{A}_{N}^{n}(\omega)\right]=[P]^{T}\left[A_{n}(\omega)\right][P],}
\end{gathered}
$$




$$
\begin{aligned}
\mathcal{F}^{n}(\omega) & =[P]^{T} \mathbf{F}(\omega) \\
\mathbf{r}_{N}^{n}(\theta, \varphi, \omega) & =[P]^{T} \mathbf{r}^{n}(\theta, \varphi, \omega)
\end{aligned}
$$

in which matrix $\left[A_{n}(\omega)\right]$ is given by Eq. (25), vector $\mathbf{F}(\omega)$ by Eq. (26) and vector $\mathbf{r}^{n}(\theta, \varphi, \omega)$ by Eq. (31). Matrix $\left[E_{n}^{N}\right]$ corresponding to $\left[E_{n}\right]$ for the reduced model is a positivesemidefinite symmetric $(n \times n)$ real matrix such that

$$
\begin{gathered}
{\left[E_{n}^{N}\right]=\int_{B}\left[e_{n}^{N}(\omega)\right] d \omega,} \\
{\left[e_{n}^{N}(\omega)\right]=[P]\left[\varepsilon_{n}^{N}(\omega)\right][P]^{T},}
\end{gathered}
$$

in which $\left[\varepsilon_{n}^{N}(\omega)\right]$ is a positive-definite symmetric $(N \times N)$ real matrix such that

$$
\left[\varepsilon_{n}^{N}(\omega)\right]=\frac{1}{\pi} \omega^{2}|\eta(\omega)|^{2} \Re e\left\{\left[\mathcal{T}_{N}^{n}(\omega)\right]^{*}[\mathcal{M}]\left[\mathcal{T}_{N}^{n}(\omega)\right]\right\}
$$

where $[\mathcal{M}]=[P]^{T}[M][P]$.

\section{Dominant eigensubspace and order of the reduced model}

In this subsection, we assume that $\mathbf{F}(\omega)=\mathbf{F}$ is independent of $\omega$ and consequently, $\mathcal{F}^{n}$ is also independent of $\omega$. Let $\mathbf{u}(\omega)$ be the solution of Eq. (12), $\mathbf{u}_{n}(\omega)$ its projection on $V_{n}$ and $\mathbf{u}_{n}^{N}(\omega)$ its projection on $V_{n}^{N} \subset V_{n}$. The energy of $\mathbf{u}_{n}$ is such that $\varepsilon_{B}\left(\mathbf{u}_{n}\right)=\sum_{\nu=1}^{n} \lambda_{\nu}^{n}\left|\mathcal{F}_{\nu}^{n}\right|^{2}$ and the energy of $\mathbf{u}_{n}^{N}$ is $\varepsilon_{B}\left(\mathbf{u}_{n}^{N}\right)=\sum_{\nu=1}^{N} \lambda_{\nu}^{n}\left|\mathcal{F}_{\nu}^{n}\right|^{2}$. We then have $\varepsilon_{B}\left(\mathbf{u}_{n}\right) \leq \lambda_{1}^{n}\left\|\mathcal{F}^{n}\right\|^{2}$ and since the upper bound is effectively reached, the maximum $\varepsilon_{\max }$ of $\varepsilon_{B}\left(\mathbf{u}_{n}\right)$ is such that $\varepsilon_{\text {max }}=\max _{\mathcal{F}^{n} \in \mathbb{C}^{n}} \varepsilon_{B}\left(\mathbf{u}_{n}\right)=\lambda_{1}^{n}\left\|\mathcal{F}^{n}\right\|^{2}$. We then deduce that $\left(\varepsilon_{B}\left(\mathbf{u}_{n}\right)-\varepsilon_{B}\left(\mathbf{u}_{n}^{N}\right)\right) / \varepsilon_{\text {max }} \leq$ $\lambda_{N+1}^{n} / \lambda_{1}^{n}$. Since $\left\{\lambda_{\nu}\right\}_{\nu}$ is a decreasing sequence of positive numbers as $\nu \rightarrow+\infty$, if $n$ is sufficiently large, then there exists $N<n$ such that $\lambda_{N+1}^{n} / \lambda_{1}^{n} \ll 1$. Subspace $V_{n}^{N}$ is called the dominant eigensubspace of operator $\mathbf{E}_{B, n}$ corresponding to the $N$ highest eigenvalues $\lambda_{1}^{n} \geq \ldots \geq \lambda_{N}^{n}$ and $N$ is called the order of the reduced model. In Sections VII and VIII, we present the graphs of the distribution of eigenvalues $\lambda_{\nu}^{n}$ for two examples which clearly show how $N$ must be chosen.

\section{CONSTRUCTION OF THE DOMINANT EIGENSUBSPACE}

All the developments and details concerning the effective construction of the dominant eigensubspace are given in Ref. 9. In order to facilitate the understanding of Sections VII and VIII and illustrate the extension necessary to take into account the presence of an external acoustic 
fluid, we summarize below the main results of the method. The reduced model defined by Eqs. (38) to (50) requires calculation of eigenvectors $\mathbf{P}^{1}, \ldots, \mathbf{P}^{N}$ in $\mathbb{R}^{n}$ corresponding to the $N$ highest eigenvalues $\lambda_{1}^{n} \geq \ldots \geq \lambda_{N}^{n}$ of the generalized symmetric eigenvalue problem defined by Eq. (37). Since $n$ is large and $N \ll n$, the Lanczos method or the subspace iteration method ${ }^{22,24-26}$ can a priori be used. The algebraic structure of matrix $\left[E_{n}\right]$ defined by Eq. (33) shows that the use of the subspace iteration method allows a very efficient solving method to be constructed, avoiding the explicit calculation of matrix $\left[E_{n}\right]$. Let $m$ be the dimension of the subspace used for the iterations such that $N<m \ll n$ (in practice ${ }^{22}$, $m=\min \{2 N, N+8\})$. Since the usual formulation of the subspace iteration method is adapted to calculating the lowest eigenvalues, Eq. (37) is transformed as follows. We have to calculate the $N$ lowest eigenvalues and associated eigenvectors of the symmetric eigenvalue problem defined by

$$
[G][S]=[H][S][\Gamma],
$$

in which $[S]$ is an $(n \times m)$ real matrix and $[\Gamma]$ a diagonal $(m \times m)$ real matrix such that $[S]^{T}[H][S]=[I]$ and $[S]^{T}[G][S]=[\Gamma]$ in which matrices $[\Gamma]$ and $[S]$ are such that $[\Lambda]=[\Gamma]^{-1}$ and $[\widetilde{P}]=[S][\Gamma]^{-1 / 2}$, where $[\Lambda]$ is the $(m \times m)$ real diagonal matrix such that the $N$ highest eigenvalues are $\lambda_{1}^{n}=[\Lambda]_{11} \geq \ldots \geq \lambda_{N}^{n}=[\Lambda]_{N N}$ and where $[\widetilde{P}]$ is the $(n \times m)$ real matrix whose first $N$ columns are eigenvectors $\mathbf{P}^{1}, \ldots, \mathbf{P}^{N}$ defining matrix $[P]$. If we examine the subspace iteration algorithm, it would seem that calculation of matrix $\left[E_{n}\right]$ is necessary. In fact, for each iteration of the algorithm, we only need to calculate an $(n \times m)$ real matrix $[W]$ such that $[W]=\left[E_{n}\right][X]$, in which $[X]$ is a given $(n \times m)$ real matrix.

\section{A. Direct procedure in the frequency domain}

The direct procedure in the frequency domain consists in calculating $\left[E_{n}\right]$ using Eqs. (33) and (34), then calculating $[W]$ each iteration ${ }^{9}$. This method is inefficient because matrix $\left[E_{n}\right]$ has to be constructed explicitly. Two other solutions are presented below.

\section{B- Indirect procedure in the frequency domain}

Since $[X]$ is a real matrix, it can easily be verified that $[W]$ can be written as $[W]=$ $\int_{B} \Re e\{[\widehat{Z}(\omega)]\} d \omega$ where $[\widehat{Z}(\omega)]$ is an $(n \times m)$ complex matrix which is the solution of

$$
\begin{aligned}
& {\left[A_{n}(\omega)\right][\widehat{Y}(\omega)]=\widehat{\chi}(\omega)[X],} \\
& {\left[A_{n}(\omega)\right][\widehat{Z}(\omega)]=[M] \overline{[\widehat{Y}(\omega)]},}
\end{aligned}
$$

J. Acoust. Soc. Am. 
in which $\widehat{\chi}(\omega)$ is a function defined on $\mathbb{R}$ such that,

$$
\widehat{\chi}(\omega)=\frac{1}{\pi} \omega^{2}|\eta(\omega)|^{2} \mathbf{1}_{B}(\omega)
$$

It is proved ${ }^{9}$ that this procedure is much more efficient than the previous one defined in Section V A.

\section{Procedure based on the use of the MF solving method in the time domain}

This method is based on the use of the MF solving method ${ }^{2,27}$ and requires factorization of only one symmetric $(n \times n)$ complex matrix. Consequently, much less core memory is necessary for this procedure than for the indirect procedure in the frequency domain (Section V B) for which $N_{\text {freq }}$ factorizations are simultaneously present in the memory, where $N_{\text {freq }}$ is the number of frequency points required for calculation of the integral in Eq. (33). A detailed analysis of this procedure is given in Ref. 9 and cannot be reproduced here. Let $\chi_{0}(t)$ be a function such that $\chi_{0}(t)=e^{-i \omega_{B} t} \chi(t)$ in which $\chi(t)=(1 / 2 \pi) \int_{B} e^{i \omega t} \widehat{\chi}(\omega) d \omega$ is the inverse Fourier transform of function $\widehat{\chi}(\omega)$ defined by Eq. (54). Therefore, the Fourier transform $\widehat{\chi}_{0}(\omega)=\int_{\mathbb{R}} e^{-i \omega t} \chi_{0}(t) d t$ is a square integrable function on $\mathbb{R}$ such that $\widehat{\chi}_{0}(\omega)=0$ if $\omega \notin B_{0}$ where $B_{0}$ denotes the LF band $B_{0}=[-\Delta \omega / 2, \Delta \omega / 2]$. Function $\chi_{0}(t)$ is the LF signal associated with the MF narrow-band signal $\chi(t)$. Let $\left[M_{B}\right],\left[D_{B}\right]$ and $\left[K_{B}\right]$ be the symmetric $(n \times n)$ matrices independent of the frequency such that $\left[M_{B}\right]=[M]+\left[B_{\Gamma}\left(\omega_{B}\right)\right]$, $\left[D_{B}\right]=\left[D\left(\omega_{B}\right)\right]$ and $\left[K_{B}\right]=\left[K\left(\omega_{B}\right)\right]$. It can be proved ${ }^{9}$ that $[W]=\left[E_{n}\right][X]$ can be calculated by $[W]=2 \pi \Re e\left\{\left[Z_{0}(0)\right]\right\}$ in which $\left[Z_{0}(t)\right]$ is the solution of the following $\mathrm{LF}$ equations in the time domain associated with the MF equations,

$$
\begin{aligned}
{\left[M_{B}\right]\left[\ddot{Y}_{0}(t)\right]+\left[\widetilde{D}_{B}\right]\left[\dot{Y}_{0}(t)\right]+\left[\widetilde{K}_{B}\right]\left[Y_{0}(t)\right] } & =\chi_{0}(t)[X], \\
{\left[M_{B}\right]\left[\ddot{Z}_{0}(t)\right]+\left[\widetilde{D}_{B}\right]\left[\dot{Z}_{0}(t)\right]+\left[\widetilde{K}_{B}\right]\left[Z_{0}(t)\right] } & =[M] \overline{\left[Y_{0}(-t)\right]},
\end{aligned}
$$

in which the symmetric $(n \times n)$ complex matrices $\left[\widetilde{D}_{B}\right]$ and $\left[\widetilde{K}_{B}\right]$ are written as $\left[\widetilde{D}_{B}\right]=$ $\left[D_{B}\right]+2 i \omega_{B}[M]$ and $\left[\widetilde{K}_{B}\right]=-\omega_{B}^{2}[M]+i \omega_{B}\left[D_{B}\right]+\left[K_{B}\right]$. It should be noted that the Fourier transforms $\left[\widehat{Y}_{0}(\omega)\right]$ and $\left[\widehat{Z}_{0}(\omega)\right]$ of $\left[Y_{0}(t)\right]$ and $\left[Z_{0}(t)\right]$ respectively, are such that $\left[\widehat{Y}_{0}(\omega)\right]=\left[\widehat{Z}_{0}(\omega)\right]=[0]$ if $\omega \notin B_{0}$. That is why Eqs. (55) and (56) are called the LF equations in the time domain associated with the MF equations related to MF band $B$. The associated LF Eqs. (55) and (56) are solved using an unconditionally stable implicit step-bystep integration method such as the Newmark method or the Wilson $\theta$ method $^{22}$. Since Eqs. 
(55) and (56) have the same differential operator $\left[M_{B}\right] d^{2} / d t^{2}+\left[\widetilde{D}_{B}\right] d / d t+\left[\widetilde{K}_{B}\right]$, only one symmetric $(n \times n)$ complex matrix has to be factorized. The basic sampling time step denoted as $\tau$ is given by Shannon's theorem and consequently, $\tau=2 \pi / \Delta \omega$. The integration time step of the step-by-step integration method is then written as $\Delta t=\tau / \mu$ where $\mu>1$ is an integer. Since $\chi_{0}$ and $\left[Y_{0}\right]$ are square integrable functions, for any $\varepsilon>0$, there exists an initial time $t_{I}=-I_{0} \times \tau$ where $I_{0}>1$ is an integer and a final time $t_{F}=J_{0} \times \tau$ where $J_{0}>1$ is another integer, such that

$$
\int_{-\infty}^{t_{I}}\left|\chi_{0}(t)\right|^{2} d t \leq \varepsilon\left\|\chi_{0}\right\|_{L^{2}}^{2} \quad, \quad \int_{t_{F}}^{+\infty}\left\|\left[Y_{0}(t)\right]\right\|^{2} d t \leq \varepsilon \int_{t_{I}}^{t_{F}}\left\|\left[Y_{0}(t)\right]\right\|^{2} d t \quad,
$$

in which $\left\|\left[Y_{0}(t)\right]\right\|^{2}=\operatorname{tr}\left\{\left[Y_{0}(t)\right]^{*}\left[Y_{0}(t)\right]\right\}$. We have the following procedure.

Step 1. Construction of the sequence $\left[Y_{0}(i \times \Delta t)\right]$ for $i=-I, \ldots, J$ with $I=I_{0} \times \mu$ and $J=J_{0} \times \mu$ by solving Eq. (55) for $\left.\left.t \in\right] t_{I}, t_{F}\right]$ with the initial conditions $\left[Y_{0}\left(t_{I}\right)\right]=$ $\left[\dot{Y}_{0}\left(t_{I}\right)\right]=[0]$.

Step 2. Construction of the sequence $\left[Z_{0}(j \times \Delta t)\right]$ for $j=-J, \ldots, 0$ by solving Eq. (56) for $\left.t \in]-t_{F}, 0\right]$ with the initial conditions $\left[Z_{0}\left(-t_{F}\right)\right]=\left[\dot{Z}_{0}\left(-t_{F}\right)\right]=[0]$.

As explained in Ref. 9, this procedure can be much more efficient than the previous one.

\section{TIME-STATIONARY RANDOM RESPONSE USING THE REDUCED MODEL}

In this section, the structural-acoustic system is submitted to a time-stationary second-order centered random wall pressure field $\{p(\mathbf{x}, t), \mathbf{x} \in \Gamma, t \in \mathbb{R}\}$ with values in $\mathbb{R}$ and we are interested in the stationary response of the structural-acoustic system. The cross-correlation function of random field $p$ is denoted as $R_{p}(\mathbf{x}, \mathbf{y}, \tau)=\mathbb{E}\{p(\mathbf{x}, t+\tau) p(\mathbf{y}, t)\}$ in which $\mathbb{E}$ is the mathematical expectation and is such that

$$
R_{p}(\mathbf{x}, \mathbf{y}, \tau)=\int_{\mathbb{R}} e^{i \omega \tau} S_{p}(\mathbf{x}, \mathbf{y}, \omega) d \omega
$$

in which $S_{p}(\mathbf{x}, \mathbf{y}, \omega)$ is the cross-spectral density function which is written as

$$
S_{p}(\mathbf{x}, \mathbf{y}, \omega)=|\eta(\omega)|^{2} s_{p}(\mathbf{x}, \mathbf{y}, \omega)
$$

\section{A. Finite dimension approximation}

Let $\left\{\mathbb{F}_{\alpha}(t), t \in \mathbb{R}\right\}$ be the stochastic process defined by

$$
\mathbb{F}_{\alpha}(t)=-\int_{\Gamma} p(\mathbf{x}, t) \mathbf{n}_{\mathbf{x}} \cdot \boldsymbol{\psi}_{\alpha}(\mathbf{x}) d s_{\mathbf{x}}
$$


Therefore the $(n \times n)$ matrix-valued spectral density function $\left[S_{\mathbb{F}}(\omega)\right]$ of stationary stochastic process $\mathbb{F}=\left(\mathbb{F}_{1}, \ldots, \mathbb{F}_{n}\right)$ indexed by $\mathbb{R}$ with values in $\mathbb{R}^{n}$ is such that

$$
\left[S_{\mathbb{F}}(\omega)\right]_{\alpha \beta}=\int_{\Gamma} \int_{\Gamma}|\eta(\omega)|^{2} s_{p}(\mathbf{x}, \mathbf{y}, \omega) \mathbf{n}_{\mathbf{x}} \cdot \boldsymbol{\psi}_{\alpha}(\mathbf{x}) \mathbf{n}_{\mathbf{y}} \cdot \boldsymbol{\psi}_{\alpha}(\mathbf{y}) d s_{\mathbf{x}} d s_{\mathbf{y}}
$$

From Eq. (24), we deduce that the $(n \times n)$ matrix-valued spectral density function $\left[S_{\mathbf{q}}(\omega)\right]$ of the second-order stationary stochastic process $\{\mathbf{q}(t), t \in \mathbb{R}\}$ with values in $\mathbb{R}^{n}$ is such that $^{28-30}$

$$
\left[S_{\mathbf{q}}(\omega)\right]=\left[T_{n}(\omega)\right]\left[S_{\mathbb{F}}(\omega)\right]{\overline{\left[T_{n}(\omega)\right.}}^{T}
$$

Below, we assume that $\theta$ and $\varphi$ are fixed in $[0,2 \pi]$ and $[0, \pi]$ respectively. The autocorrelation function $R_{c^{n}}(\theta, \varphi ; \tau)=\mathbb{E}\left\{c^{n}(\theta, \varphi, t+\tau) c^{n}(\theta, \varphi, t)\right\}$ of the second-order stationary stochastic process $\left\{c^{n}(\theta, \varphi, t), t \in \mathbb{R}\right\}$ with values in $\mathbb{R}$, corresponding to the quantity $c^{n}(\theta, \varphi, \omega)$ defined by Eq. (30), is written as

$$
R_{c^{n}}(\theta, \varphi ; \tau)=\int_{\mathbb{R}} e^{i \omega \tau} S_{c^{n}}(\theta, \varphi ; \omega) d \omega
$$

Its power spectral density function $S_{c^{n}}(\theta, \varphi ; \omega) \geq 0$ is given by ${ }^{28-30}$

$$
S_{c^{n}}(\theta, \varphi ; \omega)=\omega^{2}\left(\left[T_{n}(\omega)\right] \mathbf{r}^{n}(\theta, \varphi, \omega)\right)^{T}\left[S_{\mathbb{F}}(\omega)\right] \overline{\left[T_{n}(\omega)\right] \mathbf{r}^{n}(\theta, \varphi, \omega)}
$$

\section{B. Reduced model}

Using the reduced model defined in Section IV C, the $(n \times n)$ matrix-valued spectral density function $\left[S_{\mathbf{q}}(\omega)\right]$ of the stationary stochastic process $\mathbf{q}(t)=[P] \boldsymbol{\theta}^{n}(t)$ is written as

$$
\left[S_{\mathbf{q}}(\omega)\right]=[P]\left[S_{\boldsymbol{\theta}^{n}}(\omega)\right][P]^{T},
$$

in which the $(N \times N)$ matrix-valued spectral density function $\left[S_{\boldsymbol{\theta}^{n}}(\omega)\right]$ of the reduced model is written as $28-30$

$$
\left[S_{\boldsymbol{\theta}^{n}}(\omega)\right]=\left[\mathcal{T}_{N}^{n}(\omega)\right]\left[S_{N}(\omega)\right]{\overline{\left[\mathcal{T}_{N}^{n}(\omega)\right.}}^{T}
$$

in which the $(N \times N)$ matrix $\left[S_{N}(\omega)\right]$ is such that

$$
\left[S_{N}(\omega)\right]=[P]^{T}\left[S_{\mathbb{F}}(\omega)\right][P]
$$


The autocorrelation function $R_{c_{N}^{n}}(\theta, \varphi ; \tau)=\mathbb{E}\left\{c_{N}^{n}(\theta, \varphi, t+\tau) c_{N}^{n}(\theta, \varphi, t)\right\}$ of the secondorder stationary stochastic process $\left\{c_{N}^{n}(\theta, \varphi, t), t \in \mathbb{R}\right\}$ with values in $\mathbb{R}$, corresponding to the quantity $c_{N}^{n}(\theta, \varphi, \omega)$ defined by Eq. (42), is written as

$$
R_{c_{N}^{n}}(\theta, \varphi ; \tau)=\int_{\mathbb{R}} e^{i \omega \tau} S_{c_{N}^{n}}(\theta, \varphi ; \omega) d \omega
$$

Its power spectral density function $S_{c_{N}^{n}}(\theta, \varphi ; \omega) \geq 0$ is given by ${ }^{28-30}$

$$
S_{c_{N}^{n}}(\theta, \varphi ; \omega)=\omega^{2}\left(\left[\mathcal{T}_{N}^{n}(\omega)\right] \mathbf{r}_{N}^{n}(\theta, \varphi, \omega)\right)^{T}\left[S_{N}(\omega)\right] \overline{\left[\mathcal{T}_{N}^{n}(\omega)\right] \mathbf{r}_{N}^{n}(\theta, \varphi, \omega)}
$$

\section{VALIDATION FOR A STRUCTURE COUPLED WITH A LIQUID}

This first example concerns an inhomogeneous continuous structure immersed in a liquid (water).

\section{A. Description of the structural-acoustic system}

The structure is a thin circular cylindrical shell referred to an $r, \theta, x_{3}$ coordinate system and coupled with springs and dashpots as shown in Fig. 2. The shell is homogeneous and isotropic, with length $L=1.7 \mathrm{~m}$, constant thickness $h=0.025 \mathrm{~m}$, radius $a=0.5 \mathrm{~m}$, simply supported at its ends located at $x_{3}=0$ and $x_{3}=L$, mass density $\rho=7850 \mathrm{~kg} / \mathrm{m}^{3}$, Young's modulus $E=2.1 \times 10^{11} \mathrm{~N} / \mathrm{m}^{2}$, Poisson's coefficient $\nu=0.3$, constant damping rate $\xi=0.001$. The area $S$ of the shell surface $\Gamma$ is $S=2 \pi a L$. The ring frequency of the shell is $1726 \mathrm{~Hz}$. This cylindrical shell is coupled with 16 dashpots and 16 springs acting radially direction to the shell and located at points $\left\{a, \theta_{j}, x_{3, j}\right\}$ on surface $\Gamma$. The $j$-th dashpot has a damping coefficient $d_{j}=2 \varepsilon_{j}^{D} \mu \xi_{r e f} \omega_{r e f}$ and the $j$-th spring has a stiffness coefficient $k_{j}=2 \varepsilon_{j}^{K} \mu \omega_{r e f}^{2}$ in which $\mu=\rho h S$ is the total mass of the shell, $\xi_{\text {ref }}=0.001, \omega_{\text {ref }}=2 \pi \times 3750 \mathrm{rad} / \mathrm{s}$ and where $\varepsilon_{j}^{D}$ and $\varepsilon_{j}^{K}$ are dimensionless coefficients whose values are defined in Table I. We assume that the cylindrical shell is extended by two semi-infinite, rigid cylindrical baffles and is immersed in an unbounded liquid with a constant mass density $\rho_{E}=1000 \mathrm{~kg} / \mathrm{m}^{3}$ and speed of sound $c_{E}=1500 \mathrm{~m} / \mathrm{s}$. The coincidence frequency of the shell is $9152 \mathrm{~Hz}$ (i.e. $k a=19.2$ with $\left.k=\omega / c_{E}\right)$. We consider the MF response of this structural-acoustic system on the narrow MF band $B=2 \pi \times[4300,4800] \mathrm{rad} / \mathrm{s}$ corresponding to the $[9.00,10.05] k a$-band and we assume that for all $\omega$ in $B,|\eta(\omega)|=1$. We are only interested in calculation of the symmetric predominantly radial response of the shell. The excitation is the time-stationary random wall 
pressure $\{p(\mathbf{x}, t), \mathbf{x} \in \Gamma, t \in \mathbb{R}\}$ introduced in Section VI, for which the cross-spectral density function defined by Eq. (59) is such that $s_{p}\left(\theta, x_{3}, \theta^{\prime}, x_{3}^{\prime} ; \omega\right)=(a S)^{-1} \delta\left(\theta-\theta^{\prime}\right) \delta\left(x_{3}-x_{3}^{\prime}\right)$ where $\delta$ is the Dirac delta function.

\section{B. Description of the finite dimension approximation}

The family $\boldsymbol{\psi}_{1}, \ldots, \boldsymbol{\psi}_{n}$ introduced in Section IV is chosen as the first $n$ symmetric predominantly radial modes of the associated conservative uncoupled shell whose corresponding eigenfrequencies are $f_{1}<f_{2}<\ldots<f_{n}$. Using Donnel-Mushtani's theory ${ }^{31}$, the value chosen for $n$ is 409 . It was deduced from the convergence study of the structural-acoustic response over the $[500,7000] \mathrm{Hz}$ frequency band corresponding to the $[1.0,14.7] \mathrm{ka}$-band. We have $f_{1}=268 \mathrm{~Hz}$ and $f_{n}=11979 \mathrm{~Hz}$. The inner product $(\mathbf{u}, \mathbf{v})_{H}$ introduced in Section $\mathrm{I} \mathrm{C}$ is defined by

$$
(\mathbf{u}, \mathbf{v})_{H}=\int_{0}^{2 \pi} \int_{0}^{L} \mathbf{u}\left(\theta, x_{3}\right) \cdot \overline{\mathbf{v}\left(\theta, x_{3}\right)} a d \theta d x_{3}
$$

The normalization of the shell modes is such that, for all $\alpha$, we have $\left(\boldsymbol{\psi}_{\alpha}, \boldsymbol{\psi}_{\beta}\right)_{H}=S \delta_{\alpha \beta}$. Concerning the finite dimension approximation introduced in Section IV, matrices $[M]=$ $\mu[I]$ and $[G]=S[I]$ are diagonal, $[I]$ being the $(n \times n)$ unity matrix, and matrices $[D]$ and $[K]$ are independent of the frequency and are dense due to the presence of the dashpots and springs. The lowest eigenfrequency of the associated conservative structure (shell coupled with the springs) is $f_{1}^{S}=352 \mathrm{~Hz}$ (to be compared with $f_{1}=268 \mathrm{~Hz}$ ). Complex matrix $\left[B_{\Gamma}(\omega)\right]$ associated with the acoustic impedance boundary operator (see Section I C) is constructed explictly using the methodology developed by Junger and Feit ${ }^{14}$. The intermodal fluid couplings are neglected and consequently, $\left[B_{\Gamma}(\omega)\right]$ is a diagonal complex matrix which can be written as

$$
-\omega^{2}\left[B_{\Gamma}(\omega)\right]=-\omega^{2}\left[M_{\Gamma}(\omega)\right]+i \omega\left[D_{\Gamma}(\omega)\right]
$$

in which $\left[M_{\Gamma}(\omega)\right]=\Re e\left[B_{\Gamma}(\omega)\right]$ and $\left[D_{\Gamma}(\omega)\right]=-\omega \Im m\left[B_{\Gamma}(\omega)\right]$. Fig. 3 shows the graph of function

$$
\mu_{\Gamma}(\omega)=\frac{1}{a \rho_{E} S} \frac{1}{n} \operatorname{tr}\left[M_{\Gamma}(\omega)\right],
$$

and Fig. 4 shows the graph of function

$$
\sigma_{\Gamma}(\omega) \frac{1}{\rho_{E} c_{E} S} \frac{1}{n} \operatorname{tr}\left[D_{\Gamma}(\omega)\right]
$$


over the $[400,12000] \mathrm{Hz}$ frequency band corresponding to the $[0.8,25.1] \mathrm{ka}$-band (in Figs. 3 and 4 , the abscissas are expressed in $k a$ ). Complex vector $\mathbf{r}^{n}(\theta, \varphi, \omega)$ associated with the calculation of the far field radiated by the structure (see Eq. (30)) is obtained using a stationary-phase approximation $^{14}$.

\section{Constructing the reference solution on a broad frequency band}

Concerning the vibration of the shell coupled with dashpots, springs and external liquid, the reference solution on the $[500,7000] \mathrm{Hz}$ broad frequency band is obtained by constructing the mapping $\omega \mapsto \operatorname{tr}\left[e_{n}(\omega)\right]$ using Eq. (34), with a sampling frequency step $\delta \omega=2 \pi \times 4.33$ $\mathrm{rad} / \mathrm{s}$. In Eq. (34), $\left[T_{n}(\omega)\right]$ is calculated using Eq. (35) in which matrix $\left[A_{n}(\omega)\right]$ is calculated by Eq. (25). Fig. 5 shows the mapping $\omega \mapsto 10 \times \log _{10}\left(\operatorname{tr}\left[e_{n}(\omega)\right]\right)$ over the $2 \pi \times[500,7000]$ $\mathrm{rad} / \mathrm{s}$ broad frequency band corresponding to the $[1.0,14.7] \mathrm{ka}$-band. Concerning the far field radiated by the structure in the external liquid, Figs. 6 and 7 show the mapping $\omega \mapsto 10 \times \log _{10}\left(L^{-2} S_{c^{n}}(\theta, \varphi ; \omega)\right)$ over the $[1.0,14.7] k a$-band, calculated by using Eq. (64), for the normal and the oblique directions with respect to the shell defined by $\theta=0, \varphi=\pi / 2$ and $\theta=0, \varphi=\pi / 4$ respectively.

\section{Constructing the reference solution on the narrow MF band}

The reference solution on narrow MF band $B=2 \pi \times[4300,4800] \mathrm{rad} / \mathrm{s}$ is constructed as explained in Section VII C but using the frequency resolution $\delta \omega=\Delta \omega / N_{\text {freq }}$ in which $\Delta \omega=2 \pi \times 500 \mathrm{rad} / \mathrm{s}$ and $N_{\text {freq }}=300$. The graphs of functions $10 \times \log _{10}\left(\operatorname{tr}\left[e_{n}(\omega)\right]\right)$, $10 \times \log _{10}\left(L^{-2} S_{c^{n}}(0, \pi / 2 ; \omega)\right)$ and $10 \times \log _{10}\left(L^{-2} S_{c^{n}}(0, \pi / 4 ; \omega)\right)$ (corresponding to Figs. 5,6 and 7) of this reference solution on the $[9.00,10.05]$ narrow MF $k a$-band are used below (see Figs. 9,10 and 11) to evaluate the accuracy of the response constructed using the reduced model.

\section{E. Constructing the dominant eigensubspace}

In order to validate the reduced model procedure for a structure coupled with an external liquid, the direct procedure in the frequency domain described in Section V A is used to construct the dominant eigensubspace of matrix $\left[E_{n}\right]$ defined by Eq. (33). The approximation $\left[E_{n}\right] \simeq \delta \omega \sum_{j=1}^{N_{\text {freq }}}\left[e_{n}\left(\omega_{j}\right)\right]$ is used, in which the $\omega_{j}$ terms are the sampling frequencies of band $B$. The eigenvalues $\lambda_{1}^{n} \geq \lambda_{2}^{n} \geq \ldots>0$ and the corresponding eigenfuctions $\left\{\mathbf{e}_{1}^{n}, \mathbf{e}_{2}^{n}, \ldots\right\}$ of energy operator $\mathbf{E}_{B, n}$ are calculated using Eqs. (36) to (37). It should be noted that the 
procedure based on the use of the MF solving method in the time domain, which avoids the explicit calculation of matrix $\left[E_{n}\right]$ as explained in Section V C, has also been used to construct the dominant eigensubspace of matrix $\left[E_{n}\right]$. The results obtained are quite similar but we must limit the length of the present paper. We show the use of such a procedure in Section VIII. Fig. 8 shows the graph of the function $\nu \mapsto \lambda_{\nu}^{n}$ for $\nu \in\{1,2, \ldots, 50\}$. There is a strong decrease in the eigenvalues which means there exists the possibility of constructing an efficient reduced model independent of the spatial excitation of the structural-acoustic system. Fig. 8 shows that the order $N$ of the reduced model is about 25 for band $B$.

\section{F. Reduced model adapted to the narrow MF band}

In this section, we present a comparison of the reference solution constructed in Section VII $\mathrm{D}$ with the solution obtained by the reduced model constructed using the results of Section IV $\mathrm{C}$ in which eigenfunctions $\left\{\mathbf{e}_{1}^{n}, \ldots, \mathbf{e}_{N}^{n}\right\}$ are those calculated in Section VII E. For the three values 25, 30 and 40 of $N$, Figs. 9, 10 and 11 show the comparison of functions $10 \times \log _{10}\left(\operatorname{tr}\left[e_{n}(\omega)\right]\right), 10 \times \log _{10}\left(L^{-2} S_{c^{n}}(0, \pi / 2 ; \omega)\right)$ and $10 \times \log _{10}\left(L^{-2} S_{c^{n}}(0, \pi / 4 ; \omega)\right)$ (reference solution) with functions $10 \times \log _{10}\left(\operatorname{tr}\left[e_{n}^{N}(\omega)\right]\right), 10 \times \log _{10}\left(L^{-2} S_{c_{N}^{n}}(0, \pi / 2 ; \omega)\right)$ and $10 \times \log _{10}\left(L^{-2} S_{c_{N}^{n}}(0, \pi / 4 ; \omega)\right)$ (reduced model) calculated for each value of order $N$ using Eqs. (49)-(50) and (66), versus dimensionless wave number $k a$. It can be seen that the reduced model of order $N=25$ yields a good approximation.

\section{VALIDATION FOR A STRUCTURE COUPLED WITH A GAS}

This second example concerns an inhomogeneous continuous structure immersed in a gas (air).

\section{A. Description of the structural-acoustic system}

The structure is the thin circular cylindrical shell coupled with springs and dashpots defined in Section VII A (see Fig. 2) with only two differences related to the constant thickness which is $h=0.004 \mathrm{~m}$ instead of $h=0.025 \mathrm{~m}$, and reference frequency $\omega_{\text {ref }}$ which is $\omega_{\text {ref }}=2 \pi \times 1000$ $\mathrm{rad} / \mathrm{s}$ instead of $\omega_{\text {ref }}=2 \pi \times 3750 \mathrm{rad} / \mathrm{s}$. We assume that the cylindrical shell is extended by two semi-infinite, rigid cylindrical baffles and is immersed in an unbounded liquid with a constant mass density $\rho_{E}=1 \mathrm{~kg} / \mathrm{m}^{3}$ and speed of sound $c_{E}=300 \mathrm{~m} / \mathrm{s}$. The coincidence frequency of the shell is $2034 \mathrm{~Hz}$ (i.e. $k a=21.3$ with $k=\omega / c_{E}$ ). We consider the MF response of this

structural-acoustic system on the narrow MF band $B=2 \pi \times[1580,1680] \mathrm{rad} / \mathrm{s}$ corresponding to the $[16.54,17.59] k a$-band and we assume that for all $\omega$ in $B,|\eta(\omega)|=1$. As in Section 
VII, we are only interested in the calculation of the symmetric predominantly radial response of the shell. The excitation is the time-stationary random wall pressure $\{p(\mathbf{x}, t), \mathbf{x} \in \Gamma, t \in \mathbb{R}\}$ introduced in Section VII

\section{B. Description of the finite dimension approximation}

The finite dimension approximation is constructed as explained in Section VII B. The value chosen for $n$ is 432 . It was deduced from the convergence study of the structural-acoustic response over the $[100,1700] \mathrm{Hz}$ frequency band corresponding to the $[1.0,17.8] \mathrm{ka}$-band. We have $f_{1}=109 \mathrm{~Hz}$ and $f_{n}=2499 \mathrm{~Hz}$. The lowest eigenfrequency of the associated conservative structure (shell coupled with the springs) is $f_{1}^{S}=142 \mathrm{~Hz}$ (to be compared with $f_{1}=109 \mathrm{~Hz}$ ). Figs. 12 and 13 show the graphs of functions defined by Eqs. (72) and (73) respectively, over the $[50,2500] \mathrm{Hz}$ frequency band corresponding to the $[0.5,26.2] \mathrm{ka}$-band (in Figs. 12 and 13, the abscissas are expressed in $k a$ ).

\section{Constructing the reference solution on a broad frequency band}

This construction is performed as explained in Section VII C. Concerning the vibration of the shell coupled with dashpots, springs and external liquid, the reference solution on the $[100,1700] \mathrm{Hz}$ broad frequency band is obtained by constructing the mapping $\omega \mapsto \operatorname{tr}\left[e_{n}(\omega)\right]$ using Eq. (34), with a sampling frequency step $\delta \omega=2 \pi \times 1.06 \mathrm{rad} / \mathrm{s}$. In Eq. (34), $\left[T_{n}(\omega)\right]$ is calculated using Eq. (35) in which matrix $\left[A_{n}(\omega)\right]$ is calculated by Eq. (25). Fig. 14 shows the mapping $\omega \mapsto 10 \times \log _{10}\left(\operatorname{tr}\left[e_{n}(\omega)\right]\right)$ over the $2 \pi \times[100,1700] \mathrm{rad} / \mathrm{s}$ broad frequency band corresponding to the $[1.0,17.8] \mathrm{ka}$-band. Concerning the far field radiated by the structure in the external liquid, Figs. 15 and 16 show the mapping $\omega \mapsto 10 \times \log _{10}\left(L^{-2} S_{c^{n}}(\theta, \varphi ; \omega)\right)$ over the $[1.0,17.8] \mathrm{ka}$-band, calculated by using Eq. (64), for the normal and the oblique directions with respect to the shell defined by $\theta=0, \varphi=\pi / 2$ and $\theta=0, \varphi=\pi / 4$ respectively.

\section{Constructing the reference solution on the narrow MF band}

The reference solution on narrow MF band $B=2 \pi \times[1580,1680] \mathrm{rad} / \mathrm{s}$ is constructed as explained in Section VIII C but using the frequency resolution $\delta \omega=\Delta \omega / N_{\text {freq }}$ in which $\Delta \omega=2 \pi \times 100 \mathrm{rad} / \mathrm{s}$ and $N_{\text {freq }}=300$. The graphs of functions $10 \times \log _{10}\left(\operatorname{tr}\left[e_{n}(\omega)\right]\right)$, $10 \times \log _{10}\left(L^{-2} S_{c^{n}}(0, \pi / 2 ; \omega)\right)$ and $10 \times \log _{10}\left(L^{-2} S_{c^{n}}(0, \pi / 4 ; \omega)\right)$ (corresponding to Figs. 14, 15 and 16) of this reference solution on the [16.54,17.59] narrow MF ka-band are used 
below (see Figs. 20,21 and 22) to evaluate the accuracy of the response constructed using the reduced model.

\section{E. Constructing the dominant eigensubspace}

Since the structure is coupled with a gas, two possibilities can be examined for constructing the dominant eigensubspace of the energy operator related to band $B$. The first one consists in using the "exact" approach as was done in the first example presented in Section VII. In this case, the construction of matrix $\left[E_{n}\right]$ is based on Eqs. (33) to (35) in which matrix $\left[A_{n}(\omega)\right]$ is defined by Eq. (25). This means that eigenfunctions $\left\{\mathbf{e}_{1}^{n} \ldots, \mathbf{e}_{N}^{n}\right\}$ of $\mathbf{E}_{B, n}$ are constructed for the energy operator of the structure coupled with the external fluid. Since the external fluid is a gas, a second possibility is to construct $\left\{\mathbf{e}_{1}^{n} \ldots, \mathbf{e}_{N}^{n}\right\}$ as the dominant eigensubspace of the energy operator of the structure in a vacuum (not coupled with the gas). In this case, the procedure is exactly the same, except that matrix $\left[A_{n}(\omega)\right]$ is not defined by Eq. (25) but is written as

$$
\left[A_{n}(\omega)\right] \simeq-\omega^{2}\left([M]+\left[B_{\Gamma}(\omega)\right]\right)+i \omega[D(\omega)]+[K(\omega)]
$$

It should be noted that Eq. (74) is only used to construct $\left\{\mathbf{e}_{1}^{n} \ldots, \mathbf{e}_{N}^{n}\right\}$ but obviously not to construct the reference solution (see Sections VIII C and VIII D) and the reduced model (see Section VIII F) for which Eq. (25) is used. For the present example, we analyzed the two above possibilities and we obtained quite similar results for the reduced model. We present the results corresponding to the second approach, i.e. using Eq. (74) instead of Eq. (25) to calculate $\left\{\mathbf{e}_{1}^{n} \ldots, \mathbf{e}_{N}^{n}\right\}$. We also compared the procedures described in Section V A with the procedure described in Section V C which avoids the explicit construction of matrix $\left[E_{n}\right]$. Below we present the results correponding to the procedure based on the use of the MF solving method in the time domain (see Section V C). Figure 17 shows the graph of function $\nu \mapsto \lambda_{\nu}^{n}$ corresponding to $\left[E_{n}\right]$ calculated using Eq. (74). There is a strong decrease in the eigenvalues which means there exists the possibility of constructing an efficient reduced model independent of the spatial excitation of the structural-acoustic system. Figure 17 shows that the order $N$ of the reduced model is about 40 for band $B$. The values of the parameters of the procedure described in Section V C are as follows. The subspace iteration algorithm (see Section V) is used with $m=48$. Each iteration, matrix $[W]=\left[E_{n}\right][X]$ defined in Section $\mathrm{V}$ is calculated using the MF solving method in the time domain described in Section V C, 
with the approximation $\left[M_{B}\right] \simeq[M]$ instead of $\left[M_{B}\right]=[M]+\left[B_{\Gamma}\left(\omega_{B}\right)\right]$ for this particular example of a structure coupled with a gas. The Newmark step-by-step integration method is used $^{22}$ with scheme parameters $\alpha=1 / 4$ and $\delta=1 / 2$. Function $\chi_{0}(t)$ is deduced from Eq. (54) in which $|\eta(\omega)|=1$ if $\omega \in B$. The parameters introduced in Step 1 and Step 2 of Section $\mathrm{V}$ C for the time-solving procedure are $\mu=6, I_{0}=4$ and $J_{0}=18$, i.e. $I=24$ and $J=108$. Figure 18 shows the graph of function $i \mapsto\left\|\left[Y_{0}(i \times \Delta t)\right]\right\|^{2}$ for $i=-I, \ldots, J$ and Figure 19 shows the graph of function $j \mapsto\left\|\left[Z_{0}(j \times \Delta t)\right]\right\|^{2}$ for $j=-J, \ldots, 0$, corresponding to the last iteration of the subspace iteration algorithm (see Step 1 and Step 2 described in Section $\mathrm{V}$ C). These two figures show that the values of parameters $I_{0}$ and $J_{0}$ are correctly chosen (a similar result is obtained for each iteration, and not only for the last one).

\section{F. Reduced model adapted to the narrow MF band}

In this section, we compare the reference solution constructed in Section VIII D with the solution obtained by the reduced model constructed using the results of Section IV $\mathrm{C}$ in which eigenfunctions $\left\{\mathbf{e}_{1}^{n}, \ldots, \mathbf{e}_{N}^{n}\right\}$ are those calculated in Section VIII E. For the two values 35 and 40 of $N$, Figs. 20, 21 and 22 show the comparison of functions $10 \times \log _{10}\left(\operatorname{tr}\left[e_{n}(\omega)\right]\right), 10 \times \log _{10}\left(L^{-2} S_{c^{n}}(0, \pi / 2 ; \omega)\right)$ and $10 \times \log _{10}\left(L^{-2} S_{c^{n}}(0, \pi / 4 ; \omega)\right)$ (reference solution) with functions $10 \times \log _{10}\left(\operatorname{tr}\left[e_{n}^{N}(\omega)\right]\right), 10 \times \log _{10}\left(L^{-2} S_{c_{N}^{n}}(0, \pi / 2 ; \omega)\right)$ and $10 \times \log _{10}\left(L^{-2} S_{c_{N}^{n}}(0, \pi / 4 ; \omega)\right)$ (reduced model) calculated for each value of order $N$ using Eqs. (49)-(50) and (66), versus dimensionless wave number $k a$. It can be seen that the reduced model of order $N=40$ yields a very good approximation.

\section{CONCLUSIONS}

A theoretical approach is presented for constructing a reduced model in the MF range in the area of structural acoustic for a general three-dimensional anisotropic, inhomogeneous, viscoelastic bounded medium with an arbitrary geometry coupled with an external acoustic fluid (gas or liquid). The boundary value problem formulated in the frequency domain and its variational formulation are presented. For a given MF band, the energy operator of the structure coupled or not with the acoustic fluid is a positive-definite symmetric operator which has a countable set of decreasing positive eigenvalues. The eigenfunctions corresponding to the highest eigenvalues (dominant eigensubspace) of the energy operator are an appropriate functional basis of the admissible function space of the displacement field of the structure 
coupled with the external acoustic fluid. This functional basis allows a reduced model of the structural-acoustic system to be constructed using the Ritz-Galerkin method. A finite dimension approximation of the continuous case is introduced in a general context (using the finite element method or not). For construction of the dominant eigensubspace of the energy operator, an efficient procedure based on the use of the subspace iteration method is proposed. It does not require explicit calculation of the energy operator. We then obtain an efficient method for constructing a reduced model in the MF range. In addition, it can easily be seen that all the results presented can be extended straightforwardly to a structure made of beams, plates and shells. Analysis of the first example devoted to coupling with an external acoustic liquid (the complete analysis cannot be presented herein) shows that if the energy operator of the structure in a vacuum is used to construct the functional basis adapted to the MF band, then the order of the reduced model is much larger than if the energy operator of the structure coupled with the liquid is considered. This is due to the "added mass effect" of the fluid (this phenomenon is also encountered for the low-frequency range for which the normal modes of the structure in a vacuum are used). Consequently, it is more efficient to use the energy operator of the structure coupled with the external acoustic fluid when the fluid is a liquid. Conversely, if the external acoustic fluid is a gas, the second example presented shows that the energy operator of the structure in a vacuum can be used to construct the functional basis adapted to the MF band. Finally, the two examples presented show that the reduced model constructed yields good results.

1 C. Soize, "Vibrations linéaires moyennes fréquences des structures élastiques anisotropes," C. R. Acad. Sc., Série II 294, 895-898 (1982).

2 C. Soize, "Medium-frequency linear vibrations of anisotropic elastic structures," La Recherche Aérospatiale (English edition) 5, 65-87 (1982).

3 J. Argyris and H. P. Mlejnek, Dynamics of Structures (North-Holland, Amsterdam, 1991).

4 R. W. Clough and J. Penzien, Dynamics of Structures (McGraw-Hill, New York, 1975).

5 A. Y. T. Leung, Dynamic Stiffness and Substructures (Springer-Verlag, New York, 1993).

6 L. Meirovitch, Computational Methods in Structural Dynamics (Sijthoff and Noordhoff, The Netherlands, 1980).

7 H. J. P. Morand and R. Ohayon, Fluid-Structure Interaction (Wiley, New York, 1995). 
8 R. Ohayon and C. Soize, Structural Acoustics and Vibration (Academic Press, San Diego, 1998).

9 C. Soize, "Reduced models in the medium-frequency range for general dissipative structuraldynamics systems," European Journal of Mechanics, A/Solids 17(3), (in press) (1998).

10 Y. C. Fung, Foundations of Solid Mechanics (Prentice Hall, Englewood Cliffs, New Jersey, 1968).

11 C. Truesdell, Mechanics of Solids, Vol. III, Theory of Viscoelasticity, Plasticity, Elastic Waves and Elastic Stability (Springer-Verlag, Berlin, 1984).

12 P. G. Ciarlet, Mathematical Elasticity, Vol.I: Three-Dimensional Elasticity (North-Holland, Amsterdam, 1988).

13 R. Dautray and J.-L. Lions, Mathematical Analysis and Numerical Methods for Science and Technology (Springer-Verlag, Berlin, 1992).

14 M. C. Junger and D. Feit, Sound, Structures and Their Interaction (Acoust. Soc. Am. Publications on Acoustics, Woodbury, 1993). Originally published by MIT Press, Cambridge, 1972.

15 A. J. Burton and G. F. Miller, “The application of integral equation methods to the numerical solution of some exterior boundary value problems," Proc. R. Soc. London Ser. A. 323, 201-210 (1971).

16 D. L. Colton and R. Kress, Integral Equation Methods in Scattering Theory (Krieger Publishing Company, Malabor, Florida, 1992).

17 J. J. Angelini and P. M. Hutin, "Exterior Neumann problem for Helmholtz equation. Problem of irregular frequencies," La Recherche Aérospatiale (English edition) 3, 43-52 (1981).

18 J. T. Oden and J. N. Reddy, Variational Methods in Theoretical Mechanics, (Springer-Verlag, New York, 1983).

19 G. Duvaut and J.-L. Lions, Inequalities in Mechanics and Physics (Springer-Verlag, Berlin, 1976).

20 T. Kato, Perturbation Theory for Linear Operators (Springer-Verlag, New York, 1966).

21 M. Reed and B. Simon, Methods of Modern Mathematical Physics. I Functional Analysis (Academic Press, New York, 1980).

22 K. J. Bathe and E. L. Wilson, Numerical Methods in Finite Element Analysis ( Prentice Hall, New York, 1976). 
O. C. Zienkiewicz and R. L. Taylor, The Finite Element Method (McGraw-Hill, New York, 1989).

24 B. N. Parlett, The Symmetric Eigenvalue Problem (Prentice Hall, Englewood Cliffs, New Jersey, 1980).

25 G. H. Golub and C. F. Van Loan, Matrix Computations, 2nd Edition (The John Hopkins University Press, Baltimore and London, 1989).

26 F. Chatelin, Eigenvalues of Matrices (Wiley, New York, 1993).

27 C. Soize, P. M. Hutin, A. Desanti, J. M. David and F. Chabas, "Linear dynamic analysis of mechanical systems in the medium frequency range," Computers and Structures 23(5), 605-637 (1986).

28 C. Soize, The Fokker-Planck Equation for Stochastic Dynamical Systems and its Explicit Steady State Solutions (World Scientific, Singapore, 1994).

29 P. Kree and C. Soize, Mathematics of Random Phenomena (Reidel, Dordrecht, 1986).

30 Y.K. Lin, Probabilistic Theory of Structural Dynamics (McGraw-Hill, New York, 1967).

31 A. Leissa, Vibration of Shells (Acoust. Soc. Am. Publications on Acoustics, Woodbury, 1993). Originally published by NASA, 1973. 


\section{LEGENDS ACCOMPANYING EACH FIGURE}

Fig. 1. Geometrical configuration of the structural-acoustic system.

Fig. 2. Finite length circular cylindrical shell coupled with springs and dashpots and immersed in an acoustic fluid.

Fig. 3. Graph of function $k a \mapsto \mu_{\Gamma}(k a)$ defined by Eq. (72) for the structure coupled with a liquid.

Fig. 4. Graph of function $k a \mapsto \sigma_{\Gamma}(k a)$ defined by Eq. (73) for the structure coupled with a liquid.

Fig. 5. Reference solution of the dynamical response of the structure coupled with a liquid: graph of function $k a \mapsto 10 \times \log _{10}\left(\operatorname{tr}\left[e_{n}(k a)\right]\right)$.

Fig. 6. Reference solution of the far field radiated in the normal direction by the structure coupled with a liquid: graph of function $k a \mapsto 10 \times \log _{10}\left(L^{-2} S_{c^{n}}(0, \pi / 2 ; \omega)\right)$.

Fig. 7. Reference solution of the far field radiated in an oblique direction by the structure coupled with a liquid: graph of function $k a \mapsto 10 \times \log _{10}\left(L^{-2} S_{c^{n}}(0, \pi / 4 ; \omega)\right)$.

Fig. 8. Reference solution: graph of function $\nu \mapsto \lambda_{\nu}^{n}$ showing the distribution of eigenvalues $\lambda_{\nu}^{n}$ of energy operator $\mathbf{E}_{B, n}$ of the structure coupled with a liquid.

Fig. 9. Reduced model of the dynamical response of the structure coupled with a liquid: comparison between function $k a \mapsto 10 \times \log _{10}\left(\operatorname{tr}\left[e_{n}(k a)\right]\right)$ (reference solution (solid line)) and function $k a \mapsto 10 \times \log _{10}\left(\operatorname{tr}\left[e_{n}^{N}(k a)\right]\right.$ ) (reduced model for $N=25$ (dashed line), for $N=30$ (dotted line) and for $N=40$ (dashdot line)).

Fig. 10. Reduced model of the far field radiated in the normal direction by the structure coupled with a liquid: comparison between function $k a \mapsto 10 \times \log _{10}\left(L^{-2} S_{c^{n}}(0, \pi / 2 ; \omega)\right)$ (reference solution (solid line)) and function $k a \mapsto 10 \times \log _{10}\left(L^{-2} S_{c_{N}^{n}}(0, \pi / 2 ; \omega)\right)$ (reduced model for $N=25$ (dashed line), for $N=30$ (dotted line) and for $N=40$ (dashdot line)).

Fig. 11. Reduced model of the far field radiated in an oblique direction by the structure coupled with a liquid: comparison between function $k a \mapsto 10 \times \log _{10}\left(L^{-2} S_{c^{n}}(0, \pi / 4 ; \omega)\right)$ (reference solution (solid line)) and function $k a \mapsto 10 \times \log _{10}\left(L^{-2} S_{c_{N}^{n}}(0, \pi / 4 ; \omega)\right)$ (reduced model for $N=25$ (dashed line), for $N=30$ (dotted line) and for $N=40$ (dashdot line)).

Fig. 12. Graph of function $k a \mapsto \mu_{\Gamma}(k a)$ defined by Eq. (72) for the structure coupled with a gas. 
Fig. 13. Graph of function $k a \mapsto \sigma_{\Gamma}(k a)$ defined by Eq. (73) for the structure coupled with a gas.

Fig. 14. Reference solution of the dynamical response of the structure coupled with a gas: graph of function $k a \mapsto 10 \times \log _{10}\left(\operatorname{tr}\left[e_{n}(k a)\right]\right)$.

Fig. 15. Reference solution of the far field radiated in the normal direction by the structure coupled with a gas: graph of function $k a \mapsto 10 \times \log _{10}\left(L^{-2} S_{c^{n}}(0, \pi / 2 ; \omega)\right)$.

Fig. 16. Reference solution of the far field radiated in an oblique direction by the structure coupled with a gas: graph of function $k a \mapsto 10 \times \log _{10}\left(L^{-2} S_{c^{n}}(0, \pi / 4 ; \omega)\right)$.

Fig. 17. Reference solution: graph of function $\nu \mapsto \lambda_{\nu}^{n}$ showing the distribution of eigenvalues $\lambda_{\nu}^{n}$ of energy operator $\mathbf{E}_{B, n}$ of the structure in a vacuum (not coupled with the fluid).

Fig. 18. Graph of function $i \mapsto\left\|\left[Y_{0}(i \times \Delta t)\right]\right\|^{2}$ corresponding to the last iteration of the subspace iteration algorithm.

Fig. 19. Graph of function $j \mapsto\left\|\left[Z_{0}(j \times \Delta t)\right]\right\|^{2}$ corresponding to the last iteration of the subspace iteration algorithm.

Fig. 20. Reduced model of the dynamical response of the structure coupled with a gas: comparison between function $k a \mapsto 10 \times \log _{10}\left(\operatorname{tr}\left[e_{n}(k a)\right]\right)$ (reference solution (solid line)) and function $k a \mapsto 10 \times \log _{10}\left(\operatorname{tr}\left[e_{n}^{N}(k a)\right]\right.$ ) (reduced model for $N=25$ (dashed line), for $N=30$ (dotted line) and for $N=40$ (dashdot line)).

Fig. 21. Reduced model of the far field radiated in the normal direction by the structure coupled with a gas: comparison between function $k a \mapsto 10 \times \log _{10}\left(L^{-2} S_{c^{n}}(0, \pi / 2 ; \omega)\right)$ (reference solution (solid line)) and function $k a \mapsto 10 \times \log _{10}\left(L^{-2} S_{c_{N}^{n}}(0, \pi / 2 ; \omega)\right.$ ) (reduced model for $N=25$ (dashed line), for $N=30$ (dotted line) and for $N=40$ (dashdot line)).

Fig. 22. Reduced model of the far field radiated in an oblique direction by the structure coupled with a gas: comparison between function $k a \mapsto 10 \times \log _{10}\left(L^{-2} S_{c^{n}}(0, \pi / 4 ; \omega)\right)$ (reference solution (solid line)) and function $k a \mapsto 10 \times \log _{10}\left(L^{-2} S_{c_{N}^{n}}(0, \pi / 4 ; \omega)\right)$ (reduced model for $N=25$ (dashed line), for $N=30$ (dotted line) and for $N=40$ (dashdot line)). 


\section{TABLE}

$\begin{array}{ccll}\theta_{j}(\mathrm{deg}) & x_{3, j}(\mathrm{~m}) & \varepsilon_{j}^{D} & \varepsilon_{j}^{K} \\ 81.81 & 0.32 & 0 & 0.1 \\ -81.81 & 0.32 & 0 & 0.1 \\ 138.33 & 0.32 & 0.1 & 0.15 \\ -138.33 & 0.32 & 0.1 & 0.15 \\ 81.81 & 0.73 & 0 & 0.1 \\ -81.81 & 0.73 & 0 & 0.1 \\ 138.33 & 0.73 & 0.1 & 0.15 \\ -138.33 & 0.73 & 0.1 & 0.15 \\ 52 & 1.24 & 0.1 & 0.11 \\ -52 & 1.24 & 0.1 & 0.11 \\ 118 & 1.24 & 0.1 & 0.14 \\ -118 & 1.24 & 0.1 & 0.14 \\ 52 & 1.55 & 0.1 & 0.11 \\ -52 & 1.55 & 0.1 & 0.11 \\ 118 & 1.55 & 0.1 & 0.14 \\ -118 & 1.55 & 0.1 & 0.14\end{array}$

Table I. Values of $\varepsilon_{j}^{D}$ and $\varepsilon_{j}^{K}$ 


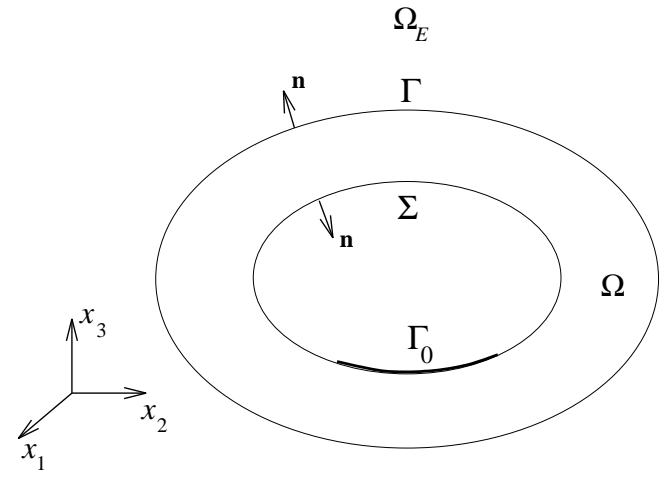

Fig. 1, Christian Soize, J. Acoust. Soc. Am.

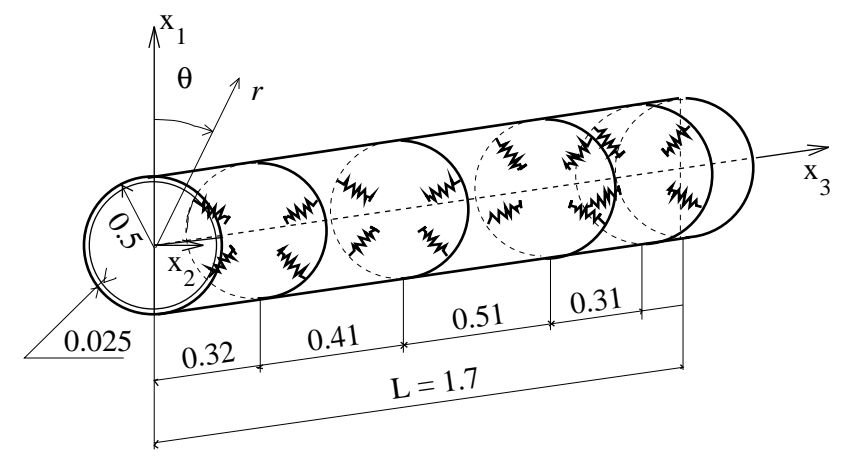

Fig. 2, Christian Soize, J. Acoust. Soc. Am.

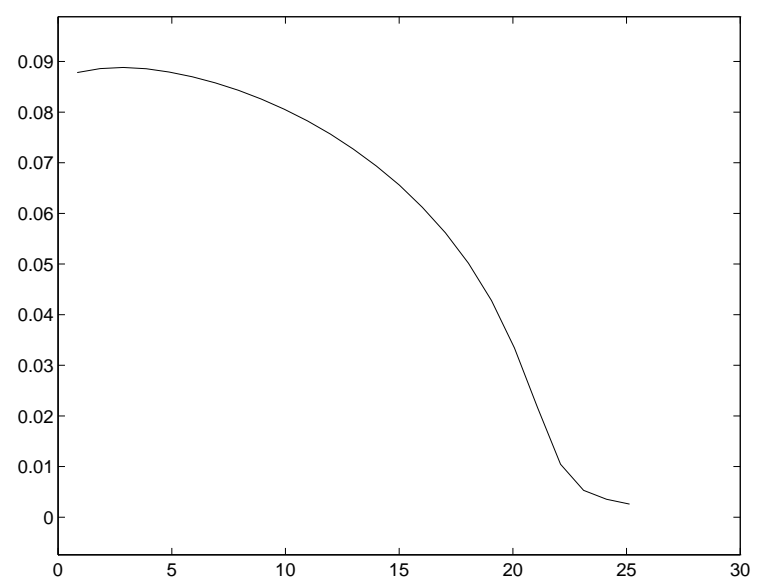

Fig. 3, Christian Soize, J. Acoust. Soc. Am.

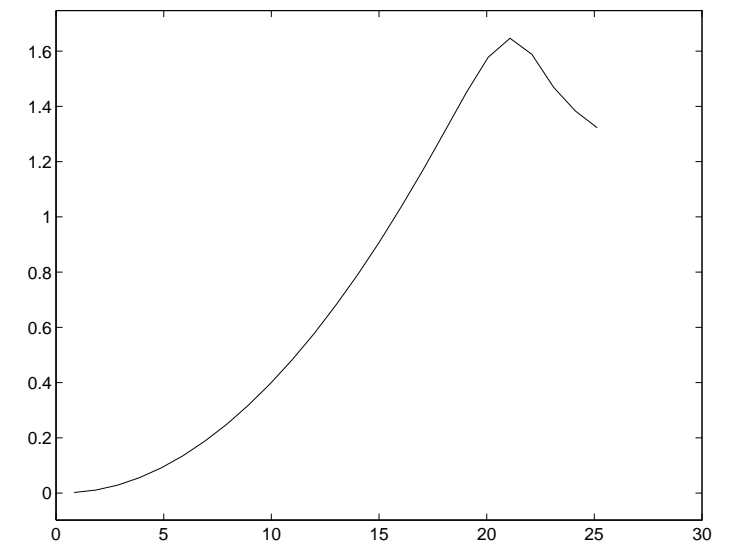

Fig. 4, Christian Soize, J. Acoust. Soc. Am.

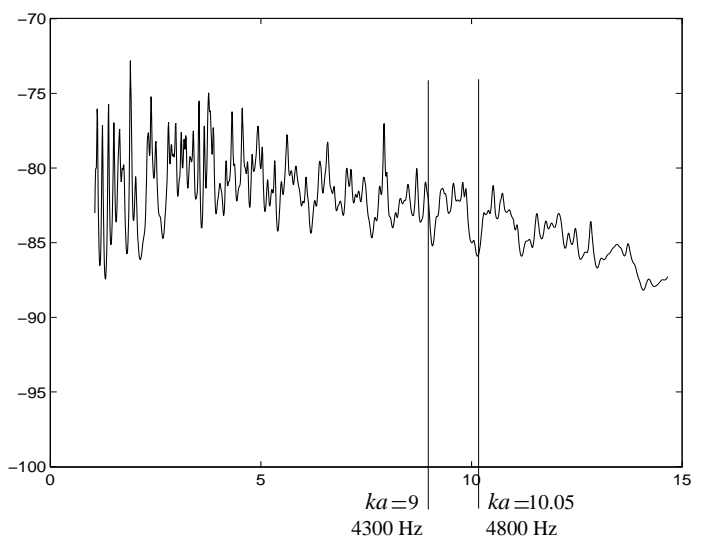

Fig. 5, Christian Soize, J. Acoust. Soc. Am.

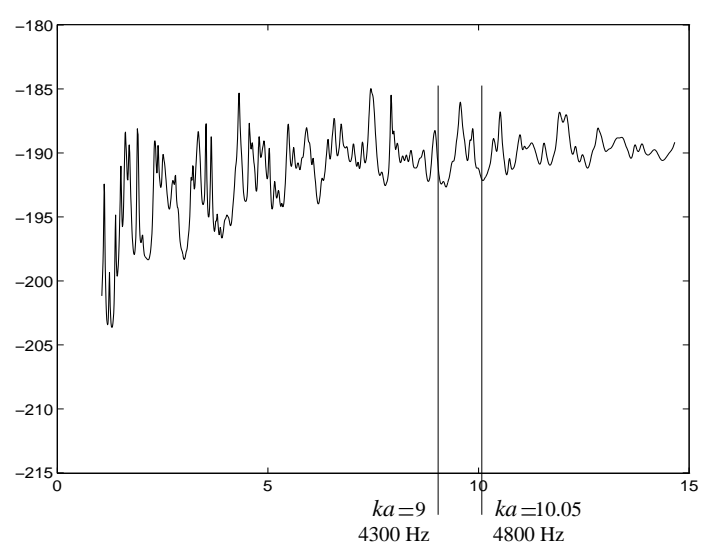

Fig. 6, Christian Soize, J. Acoust. Soc. Am. 


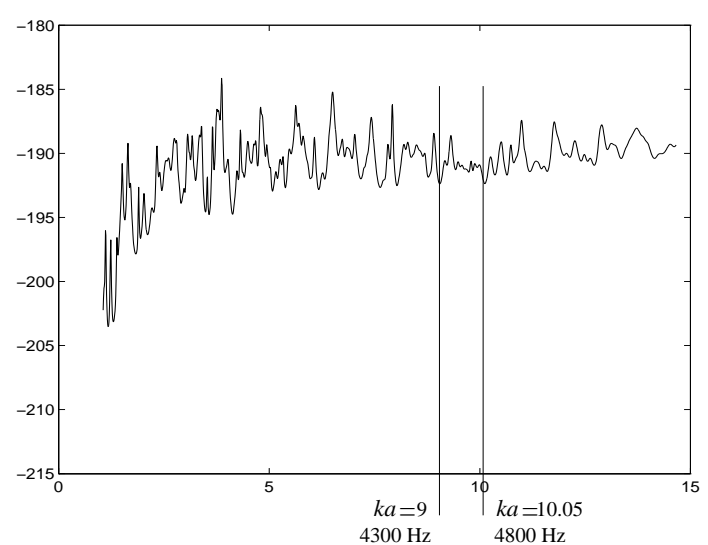

Fig. 7, Christian Soize, J. Acoust. Soc. Am.

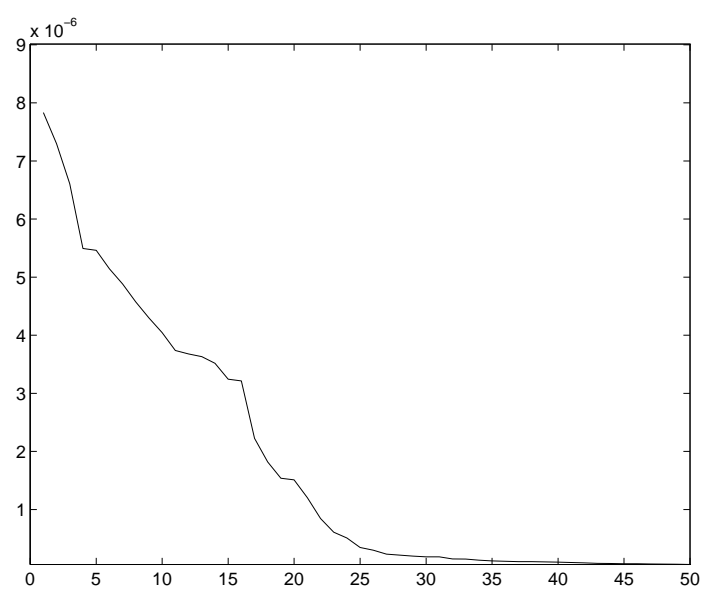

Fig. 8, Christian Soize, J. Acoust. Soc. Am.

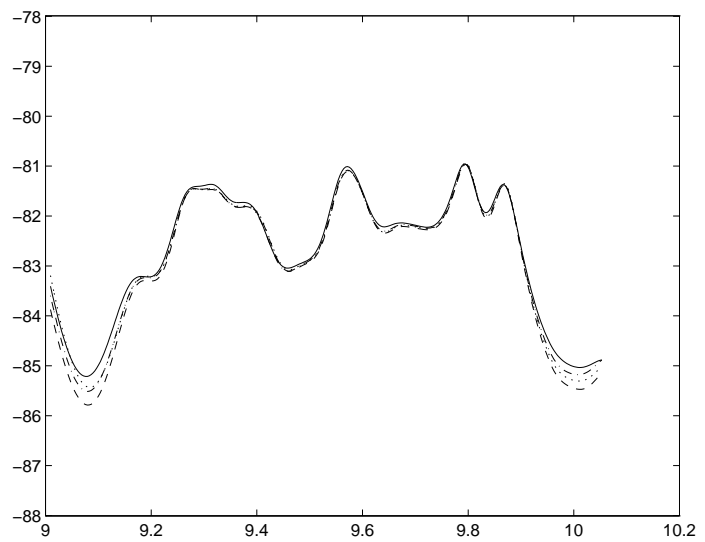

Fig. 9, Christian Soize, J. Acoust. Soc. Am.

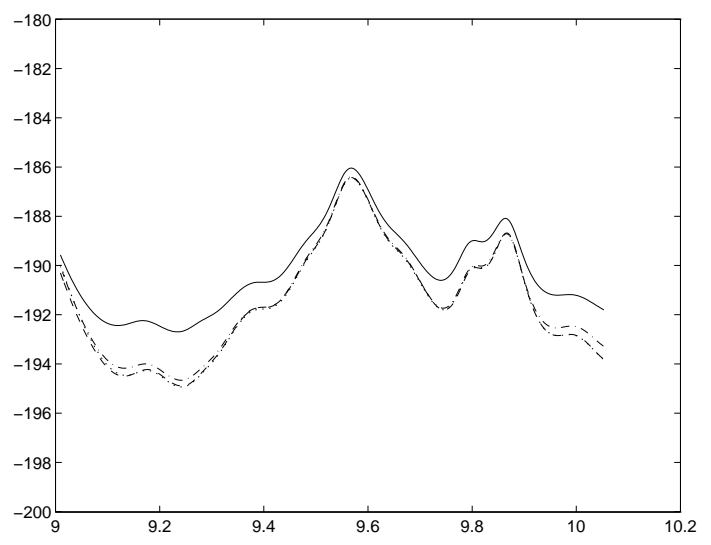

Fig. 10, Christian Soize, J. Acoust. Soc. Am.

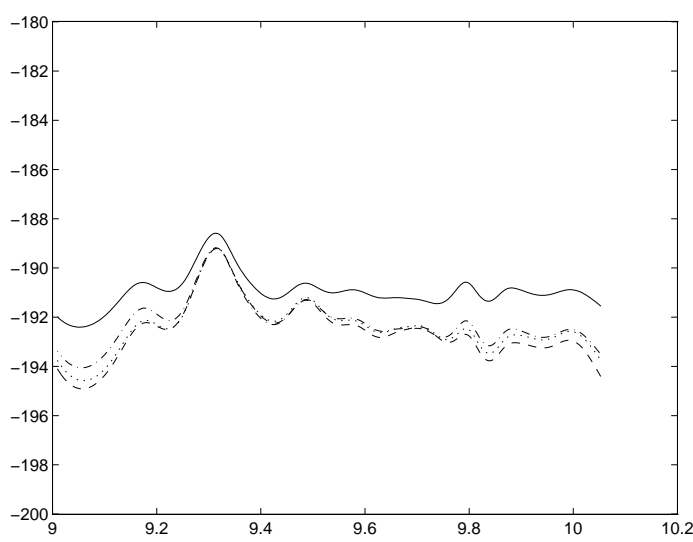

Fig. 11, Christian Soize, J. Acoust. Soc. Am.

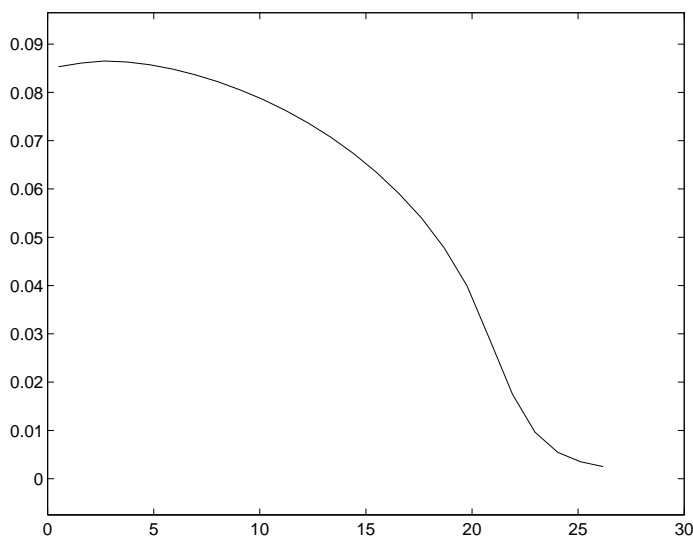

Fig. 12, Christian Soize, J. Acoust. Soc. Am. 


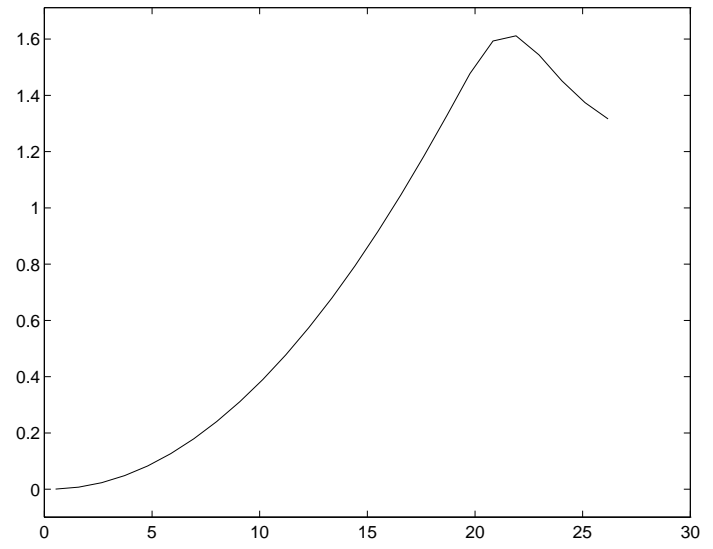

Fig. 13, Christian Soize, J. Acoust. Soc. Am.

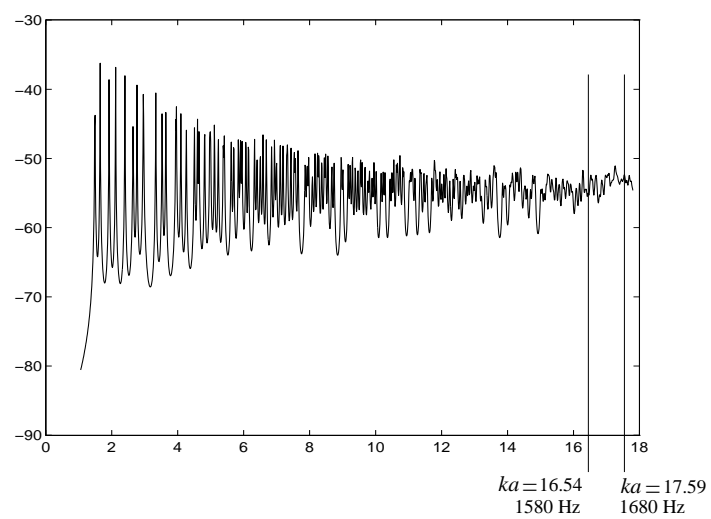

Fig. 14, Christian Soize, J. Acoust. Soc. Am.

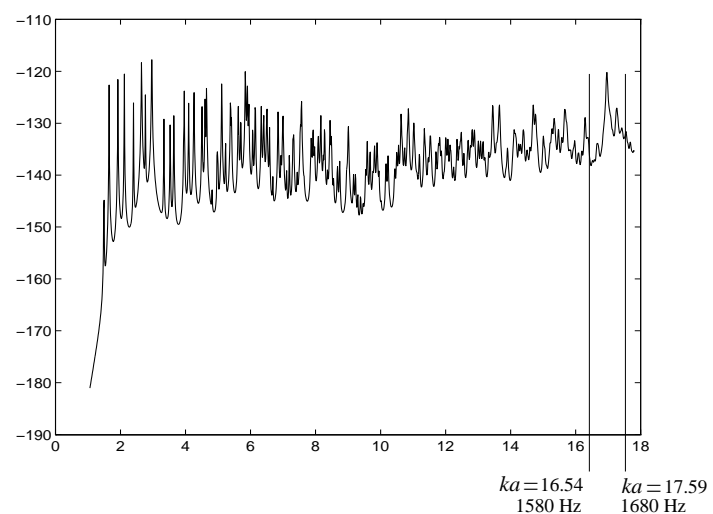

Fig. 15, Christian Soize, J. Acoust. Soc. Am.

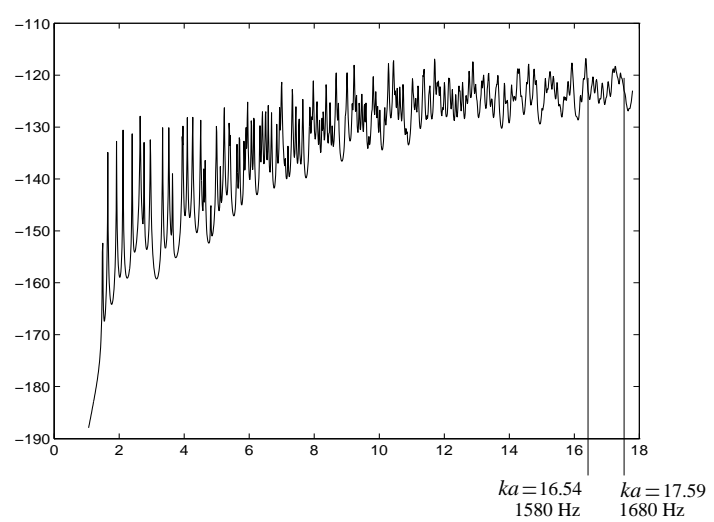

Fig. 16, Christian Soize, J. Acoust. Soc. Am.

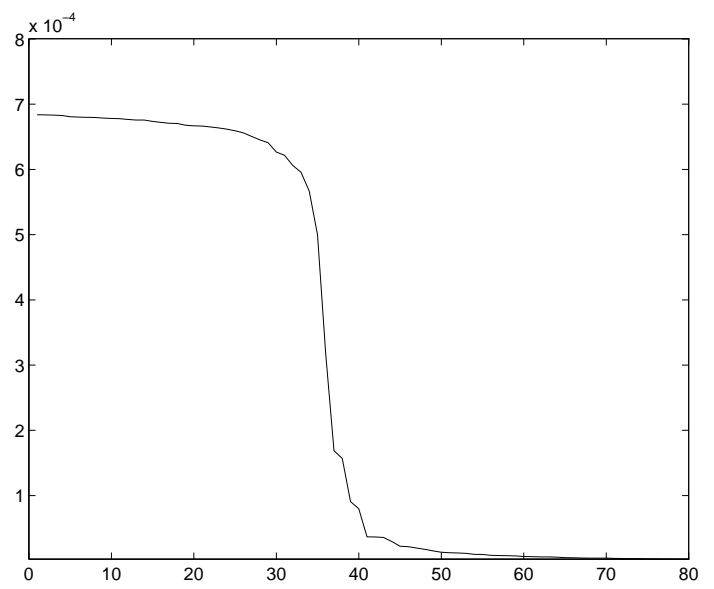

Fig. 17, Christian Soize, J. Acoust. Soc. Am.

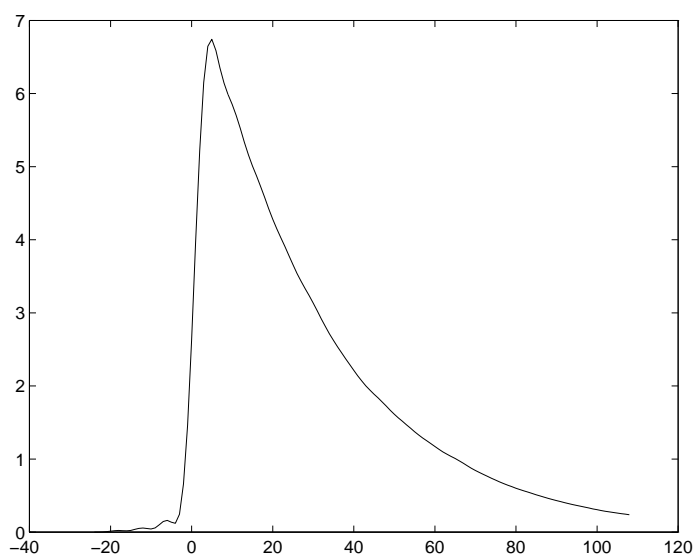

Fig. 18, Christian Soize, J. Acoust. Soc. Am. 


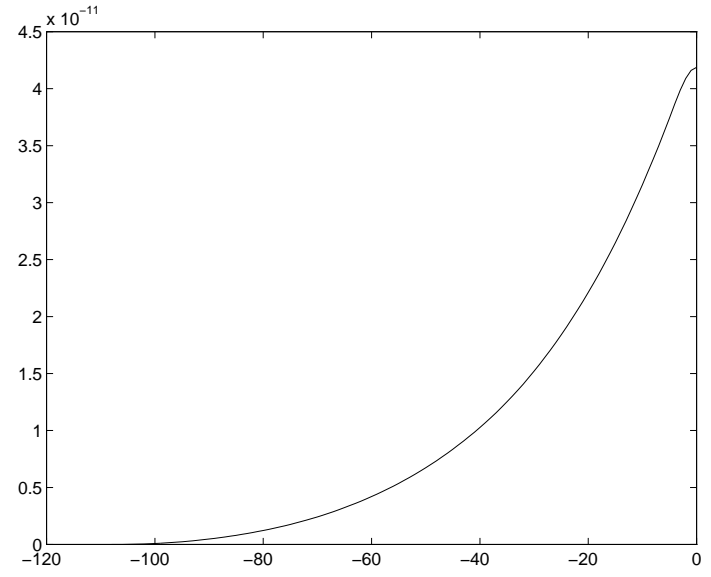

Fig. 19, Christian Soize, J. Acoust. Soc. Am.

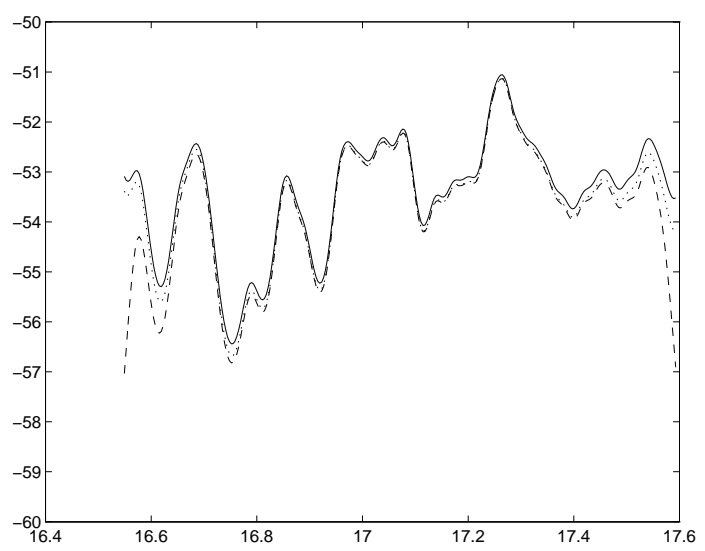

Fig. 20, Christian Soize, J. Acoust. Soc. Am.

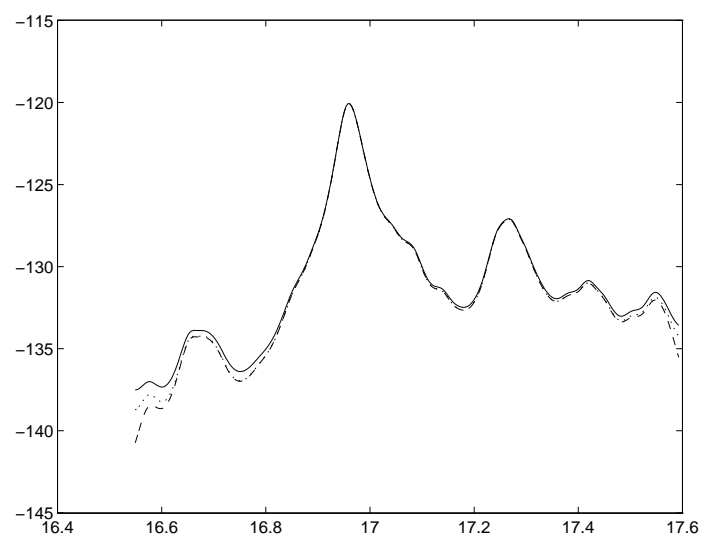

Fig. 21, Christian Soize, J. Acoust. Soc. Am.

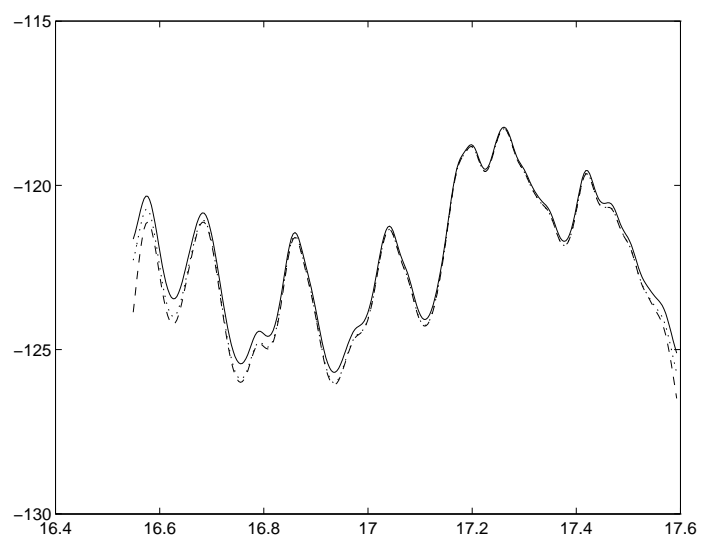

Fig. 22, Christian Soize, J. Acoust. Soc. Am. 